\title{
Low-Power, Low-Voltage Complex Gm-C Filter Structure With Self-Common-Mode Control
}

\begin{tabular}{|r|l|}
\hline Journal: & IEEE Transactions on Circuits and Systems I: Regular Papers \\
\hline Manuscript ID & Draft \\
\hline Manuscript Type: & Regular Paper \\
\hline Complete List of Authors: & $\begin{array}{l}\text { Khumsat, Phanumas; Prince of Songkla University, Department of Electrical } \\
\text { Engineering }\end{array}$ \\
\hline EDICS: & $\begin{array}{l}\text { ACS140 - Analog filters < ACS - Analog and Mixed Mode Circuits and } \\
\text { Systems, ACS130 - Amplifiers < ACS - Analog and Mixed Mode Circuits and } \\
\text { Systems, ACS120 - Low power/low voltage analog circuits < ACS - Analog } \\
\text { and Mixed Mode Circuits and Systems }\end{array}$ \\
\hline
\end{tabular}




\title{
Low-Power, Low-Voltage Complex $G_{m}-C$ Filter Structure With Self-Common-Mode Control
}

\author{
Phanumas Khumsat Member, IEEE
}

\begin{abstract}
This work develops a low-power and low-voltage differential $G_{m}-C$ filter structure that effectively achieves selfcommon-mode control (SCC), including DC stabilization and common-mode (CM) rejection, without employing extra control circuitry. The structure relies upon an incorporation of voltage inverting amplifiers to make it inherently contain no $\mathrm{CM}$ positive feedback loops for DC stabilization, and to enable splitting of the core transconductors into pairs for CM signal rejection. A DC CM stability analysis reveals that stabilization of the SCC structure can be reached without any dedicated CM control circuitry. An analytical comparison on power consumption of a high-order lowpass $G_{m}-C$ filter implemented using an inverterbased transconductor for the SCC structure and the same transconductor with a CM control network (the Nauta's technique) for the conventional structure indicates theoretical overhead power saving by over 50\%. Furthermore, an even higher overhead power saving at over $70 \%$ can be achieved in the complex SCC $G_{m}-C$ filter because no additional inverting amplifiers are required to eliminate CM positive feedback loops in the crossing transconductors for complexification. The impact of the inverting amplifiers on the noise and frequency characteristics as well as the compensation technique are outlined to enable design optimization. The SCC filter was verified via extensive simulations of a $5^{\text {th }}$-order $1.1-\mathrm{MHz}$ elliptic complex filter in a $0.18-\mu \mathrm{m}$ CMOS process. As compared to the conventional filter counterpart with similar SNR ( 63dB) and inband/out-of-band SFDRs $(\sim 52 \mathrm{~dB} / 56 \mathrm{~dB})$, the proposed structure yields an overhead power saving by $70 \%$ with an improved figure-of-merit over $40 \%$ under a 1-V supply.
\end{abstract}

Index Terms $-G_{m}-C$ filter, transconductor, Nauta, complex filter, CMOS, common-mode, stability, positive feedback

\section{INTRODUCTION}

A complex filter is one of the essential building blocks in modern wireless Low-IF receivers [1]-[13]. Its main function is to remove any image signal interference coming from adjacent radio channels after an RF-to-IF down conversion. The transconductor-capacitor $\left(G_{m}-C\right)$ complex filter has been an attractive filtering solution due to its suitability for highfrequency and low-voltage operation stemming from the transconductor's simplicity and compactness [14]-[16]. Under a very low-voltage supply $V_{D D}$, it becomes increasingly challenging to implement a MOS transconductor with the capability to reject common-mode (CM) signals and prevents

This paragraph of the first footnote will contain the date on which you submitted your paper for review. This research work has been financially supported from Thailand Research Fund (TRF), Prince of Songkla University and Faculty of Engineering under the grant number RSA5780050.

P. Khumsat is with the Department of Electrical Engineering, Faculty of Engineering, Prince of Songkla University, Hat Yai, Songkhla, 90112 Thailand (e-mail: phanumas.k@psu.ac.th).

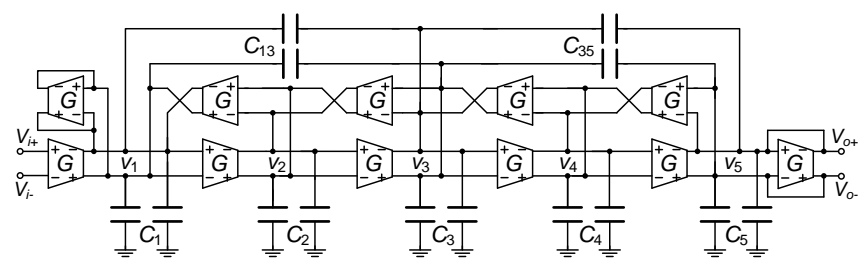

(a) Differential circuit, $G=$ differential-mode (DM) transconductance

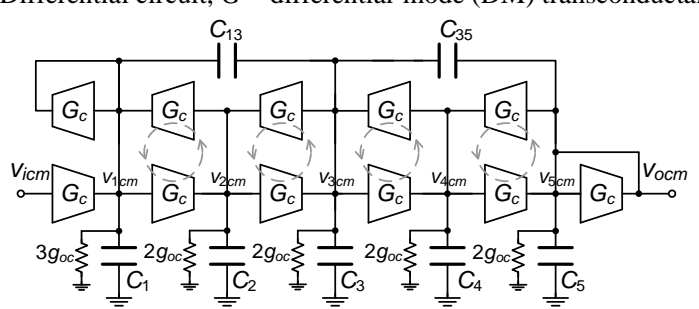

(b) $\mathrm{CM}$ equivalent circuit indicating an inherent positive feedback loop, $G_{c}$ $=\mathrm{CM}$ transconductance, $g_{o c}=\mathrm{CM}$ output conductance

Fig. 1. Conventional differential $G_{m}-C$ filter (convention: output current flow into the transconductor with a positive voltage swing)

instability arising from CM positive feedback loops inside the conventional differential $G_{m}-C$ filter structure as shown in Fig. 1(a) (there are four head-to-tail CM positive feedback loops shown in Fig. 1(b)).

A low-power, low-voltage filter design typically makes use of a pseudo-differential transconductor with $\mathrm{CM}$ control circuitry based upon common-mode feedback (CMFB), common-mode feedforward (CMFF) or commonmode/differential-mode feedforward (CM/DM-FF) techniques. In [17], a CMFB technique was used to increase the transconductor's CM output conductance, $g_{o c}$, to lower the $\mathrm{CM}$ loop gain so as to prevent instability as well as to set up a DC bias level of the source-degenerated pseudo-differential transconductor. This CMFB technique usually demands high power consumption and silicon area due to the deployment of error feedback amplifiers. Alternatively, the filters in [6], [1821] utilize $\mathrm{CMFF}$ and $\mathrm{CM} / \mathrm{DM}-\mathrm{FF}$ techniques which essentially minimize the $\mathrm{CM}$ loop gain by making the $\mathrm{CM}$ transconductance, $G_{c} \approx 0$. The transconductor with CMFF can double power consumption and transistor area [18-20], making it rather inefficient in terms of transconductancepower ratio. Moreover, such CMFF technique also requires another CMFB loop for DC bias setup. A more powerefficient solution presented in [6] utilizes both CMFB and CMFF only at the biquad's integrating nodes (outside the transconductor cell). The transconductors with CM/DM-FF in [21-23] further improve efficiency since their cross-forward structure simultaneously provides CM control and doubles DM transconductance. However, its operation is heavily dependent on transistor matching. 


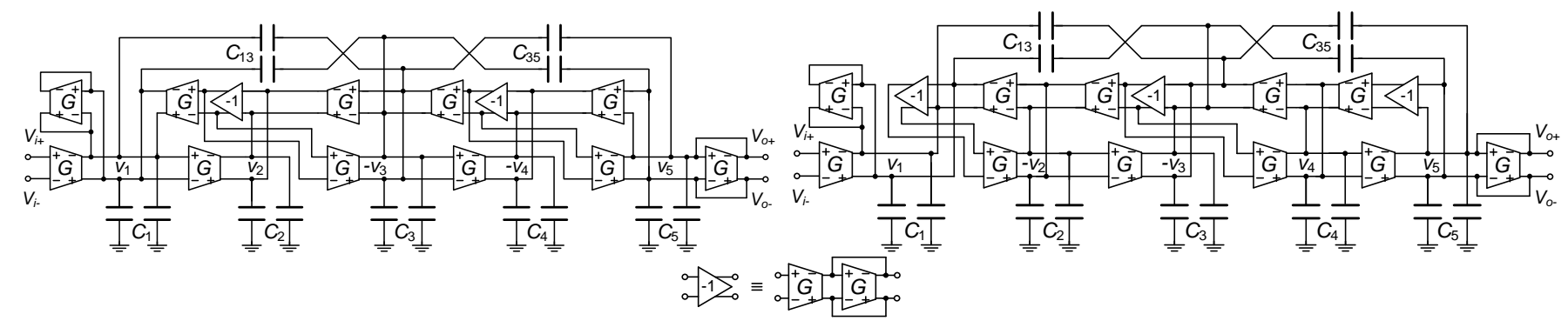

(a) Inverting amplifiers attached only at $e e^{\text {th }}$ nodes - the even type (left) and odd $d^{\text {th }}$ nodes - the odd type (right)

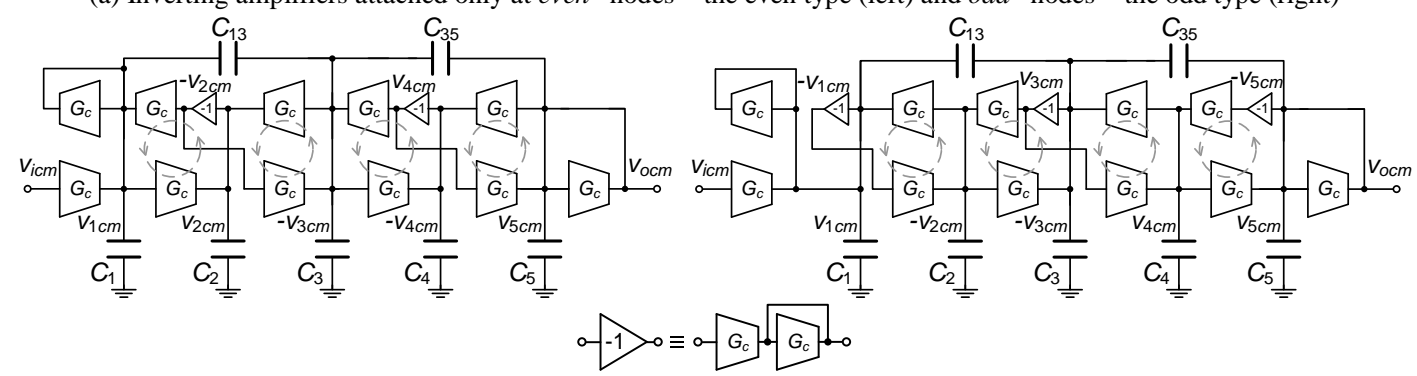

(b) Corresponding CM equivalent circuits of (a): the even type (left) and the odd type (right)

Fig. 2. Proposed differential $G_{m}$-C filters with basic self-CM-control (SCC) mechanism which inherently contain no CM positive feedback loop.

The recent current-recycling technique [24-25] offers a compact and low power $G_{m}-C$ complex filter with a very competitive figure-of-merit. This technique, however, might not be applicable to a general $G_{m}-C$ filter such as that realized from a $L C$-ladder prototype. Alternatively, the Nauta's inverter-based transconductor has been widely employed in $G_{m}-C$ filters intended for high-frequency, highly linear and large-dynamic range applications under a low-voltage supply [7], [26-37]. However, the CM control network inside the Nauta's transconductor, which equivalently sets $g_{o c}=G_{c}$ (Fig. $1(\mathrm{~b})$ ), is responsible for nearly half of the filter's total power consumption and transconductor area.

To alleviate the aforementioned problems, a powerefficient low-voltage $L C$-ladder-based differential $G_{m}-C$ complex filter structure is proposed. The structure is stabilized systematically by removing all the CM positive feedback loops typically found in the conventional structure. Without employing any CMFB or CMFF technique to maximize $g_{o c}$ or minimize $G_{c}$, the filter can be inherently CM stabilized, thereby achieving significant power reduction.

Section II.A introduces the low-voltage $G_{m^{-}} C$ filter with basic self-CM control (SCC) that can be directly realized from a $L C$-ladder lowpass filter structure. The complete SCC filter structure with CM rejection ability is developed in Section II.B as a more general filter structure. An analysis on the loop gains and nodal conductances is carried out in Section II.C, D for the filter's DC CM stability and CM gain inspection. The filter's power efficiency is assessed in Section II.E with an overhead-power ratio between the proposed and the conventional based on the Nauta's transconductor. Section III.A illustrates a low-power $5^{\text {th }}$-order SCC complex elliptic filter. An overhead-power comparison of the complex filter is estimated in Section III.B. Noise performance and frequency response influenced by non-idealities of the proposed structure are also studied with a frequency compensation method presented. The practical feasibility of the SCC filter is verified in Section IV with extensive simulations of the $5^{\text {th }}$-order 1.1$\mathrm{MHz}$ complex elliptic filter in a standard $0.18-\mu \mathrm{m}$ CMOS process.

\section{II. $G_{M}-C$ Filter Structure With Self Common-Mode CONTROL (SCC)}

\section{A. Basic SCC Structure with DC Stabilization}

Without loss of generality, the basic self-CM-control (SCC) structure will be developed from the $L C$-ladder-based $5^{\text {th }}$-order $G_{m}-C$ filter of Fig. 1(a). This is illustrated with the differential $G_{m}-C$ filter in Fig. 2(a). Without disturbing the transfer function of the conventional filter, inverting voltage amplifiers are inserted only at the $e v e n^{\text {th }}$ nodes (even type) or only at the odd ${ }^{\text {th }}$ nodes (odd type). The inverting voltage amplifier can be simply constructed from two transconductors (the bottom of Fig. 2(a)). Since there are sign alterations of $V_{3},-V_{4}$ and $-V_{2},-V_{3}$ in Fig. 2(a) as compared to the node voltages in the conventional filter, this basic SCC filter thus necessitates cross-over connections of $C_{13}$ and $C_{35}$.

The key purpose for the inclusion of the inverting amplifiers is to eliminate CM positive feedback loops normally existing in the conventional differential filter structure. This can be explained by considering the $\mathrm{CM}$ equivalent circuits of the SCC $G_{m}-C$ filter structures. As shown in Fig. 2(b), the associated CM feedback loops (dash circles in the figures) are all in a negative feedback manner. Thus, the proposed structure requires no additional circuitry to stabilize the CM voltages inside each of the transconductors. This significantly helps save power consumption and silicon area compared to the conventional structure. The extra power and area consumptions added to the core transconductors, $G$, are mainly from the inverting voltage amplifiers.

With the inclusion of the inverting amplifiers, this SCC structure might look similar to the biquadratic-based $G_{m}-C$ filter in [38]. However in this case, there is no requirement to load the output of each transconductor with a small adjustable 


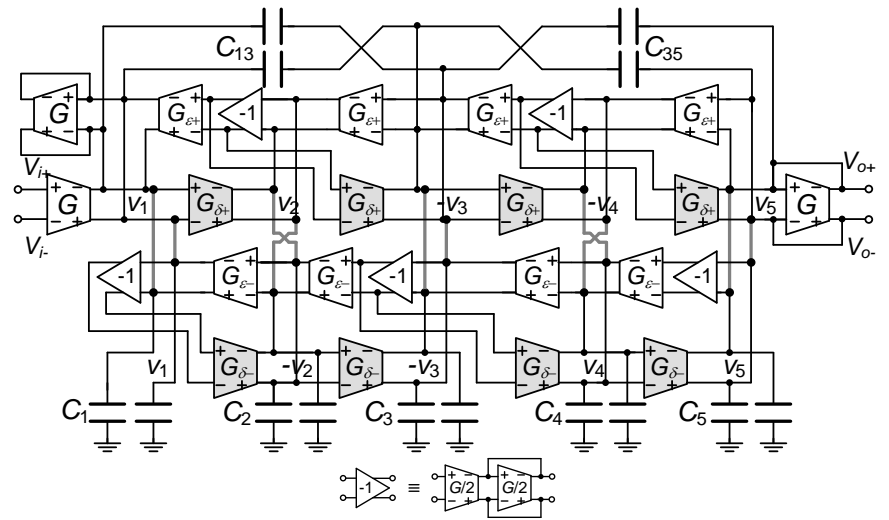

(a) Differential implementation with $G_{\varepsilon \pm}=(1 \pm \varepsilon) G / 2, G_{\delta \pm}=(1 \pm \delta) G / 2$

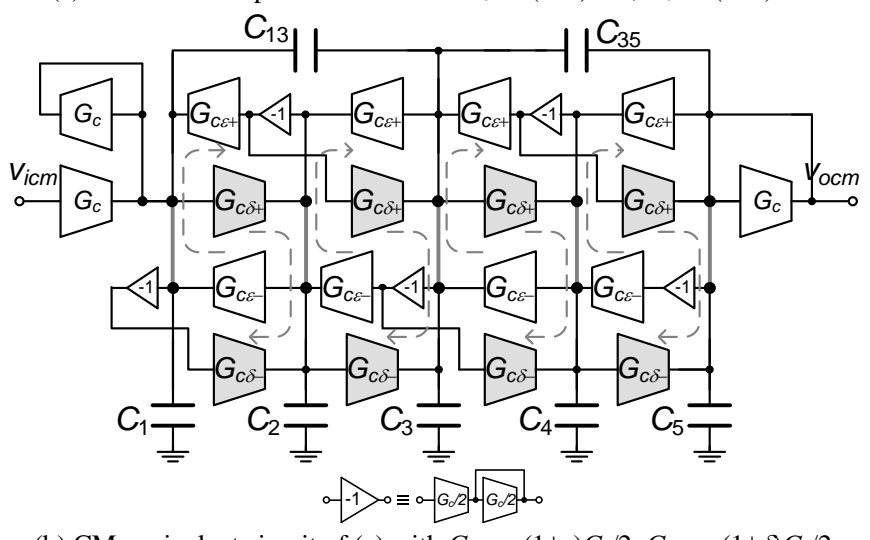

(b) $\mathrm{CM}$ equivalent circuit of (a) with $G_{c \varepsilon \pm}=(1 \pm \varepsilon) G_{c} / 2, G_{c \delta \pm}=(1 \pm \delta) G_{c} / 2$

Fig. 3. Proposed self-CM-control (SCC) $G_{m}$-C filter with CM Rejection.

$1 / G$ resistor in order to avoid using a CMFB circuit and maintain CM stability. Moreover, the basic SCC structure can be readily applied to more general $G_{m}-C$ filters - especially those realized from well-established, least-sensitive, doubly terminated $L C$-ladder filters.

\section{B. SCC Filter with CM Rejection}

By inspecting the circuits in Fig. 2, it is clear that the DC CM gain of the basic SCC lowpass filter is identical to that of the DC DM gain at $1 / 2$, and this inevitably renders a DC common-mode rejection ratio (CMRR) of unity. In order to maintain the DC-stabilizing feature and simultaneously allow $\mathrm{CM}$ rejection, the basic structures in Fig. 2(a) are modified into the complete SCC structure in Fig. 3(a) without disturbing the original DM response with the modified transconductors $G_{\delta \pm}=(1 \pm \varepsilon) G / 2$ and $G_{\delta \pm}=(1 \pm \delta) G / 2$. Note that $\varepsilon$ and $\delta$ are of the same polarity and $0<(\varepsilon, \delta) \leq 1$ or $-1 \leq(\varepsilon, \delta)<0$. This complete SCC structure, still preserves the original low-power property where the total transconductance value of the core transconductors and the inverting amplifiers remains unchanged.

When $(\varepsilon, \delta) \neq 0$, it can be seen from the corresponding CM equivalent circuit in Fig. 3(b) that the associated CM feedback loops still remain in a negative feedback manner. Moreover, there is a certain degree of CM current cancellation between the shaded transconductor pairs, $G_{\delta+}$ and $G_{\delta-}$ as well as the non-shaded pairs, between $G_{\varepsilon+}$ and $G_{\varepsilon-\text { }}$, due to the presence of the inverting amplifiers. By inspecting the DM and CM signal excursions inside the $G_{\varepsilon \pm}$ pairs and the $G_{\delta \pm}$ pairs, it suggests that these transconductor pairs basically resembles CM/DMFF operation (with shared inverting amplifiers) at the structural level as compared to the circuit-level implementation found in [21-23]. Such similarity renders the forward CM transconductance from $j^{\text {th }}$ node to $(j+1)^{\text {th }}$ node, $G_{c f w d}=\delta G_{c}$, and the backward CM transconductance from $j^{\text {th }}$ to $(j-1)^{\text {th }}$ node, $G_{c b k w d}=\varepsilon G_{c}$.

If $\varepsilon=\delta=0$, one may hope to enjoy a perfect CM rejection. However, since all the filter's internal nodes (except the first and last nodes) becomes a DC CM open circuit (zero conductance) and the filter stays right at the borderline between DC stable and unstable regions. This issue will become clearer with the $\mathrm{CM}$ analysis presented in the following subsection. Specifically for $\varepsilon=\delta=+1(-1)$, the transconductors $G_{\varepsilon^{-}}, G_{\delta-}\left(G_{\delta_{+}}, G_{\delta_{+}}\right)$would varnish and the inverting amplifiers associated with them become redundant. Thus the structure is simply reduced to the original even-type (odd-type) basic SCC filter of Fig. 2(a). Therefore the complete SCC filter is a more general structure that covers both odd- and even-type basic SCC filters.

\section{DC and Common-Mode Stability Inspection}

Fig. 4(a) shows the DC CM equivalent circuit of the proposed SCC filter structure for DC stability verification via an analysis of the DC loop gains. To enable the use of the analysis for DC stability discussion on the conventional filters with other $\mathrm{CM}$ control techniques, the CM output conductance $g_{o c}$ of each $G_{c}$ (except the input $G_{c i}$ for ease of analysis) is also included. The factor $\gamma$ represents the inverting amplifier's voltage gain which may be different from -1 . The parameters $G_{j}$ and $\hat{G}_{j}$ are the DC CM conductance looking from the $j^{\text {th }}$ node to the right side and the left side, respectively. According to these conductance definitions, it follows that $\hat{G}_{1}=G_{N}=$ $2 g_{o c}+G_{c}$, and $G_{j}$ for $j=N-1, N-2 \ldots, 1$ can be calculated recursively as

$G_{j}=2 g_{o c}-\frac{G_{c}^{2}}{4 G_{j+1}}[(1+\gamma) \pm \delta(1-\gamma)][(1+\gamma) \mp \varepsilon(1-\gamma)]$.

Similarly, $\hat{G}_{j}$ for $j=2,3 \ldots, N$ can be expressed as

$\hat{G}_{j}=2 g_{o c}-\frac{G_{c}^{2}}{4 \hat{G}_{j-1}}[(1+\gamma) \pm \varepsilon(1-\gamma)][(1+\gamma) \mp \delta(1-\gamma)]$.

Similar to the analysis in [39], the effective DC loop gain $L G_{j}$ at the $j^{\text {th }}$ node, for $j=1,2, \ldots, N$ in Fig. 4(a), can be derived in terms of the $j^{\text {th }}$ conductances from the two possible feedback formations in Fig. 4(b), and their corresponding current feedback system in Fig. 4(c) as

$$
L G_{j}=2-\left(G_{j}+\hat{G}_{j}\right) / 2 g_{o c} .
$$

It is known that a negative feedback loop gain indicates stability regardless of its magnitude. On the other hand, a positive loop gain can indicate stability only if its magnitude is less than unity. For the case of multiple loops such as the CM DC equivalent circuit of Fig. 4(a), all the loop gains $L G_{j}$ must be less than unity to indicate overall DC stability, i.e., $L G_{j}<$ +1 for all $j=1$ to $N$. 

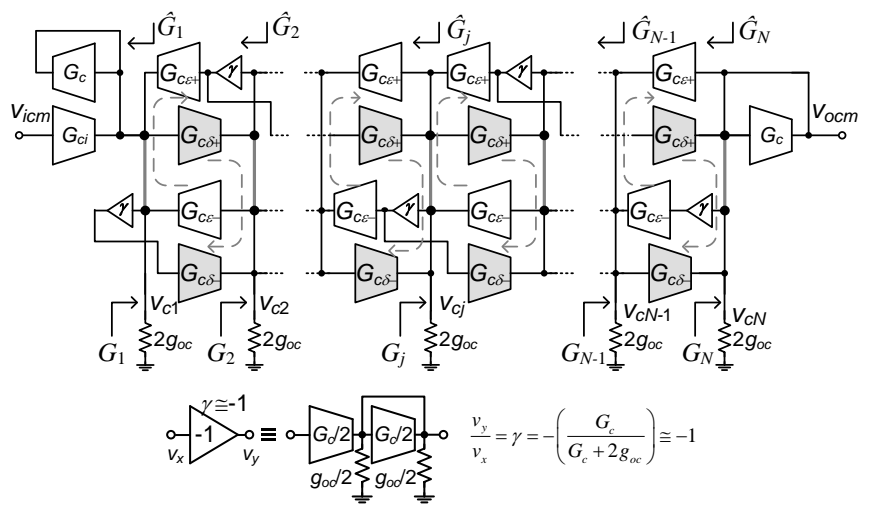

(a) DC CM equivalent circuit
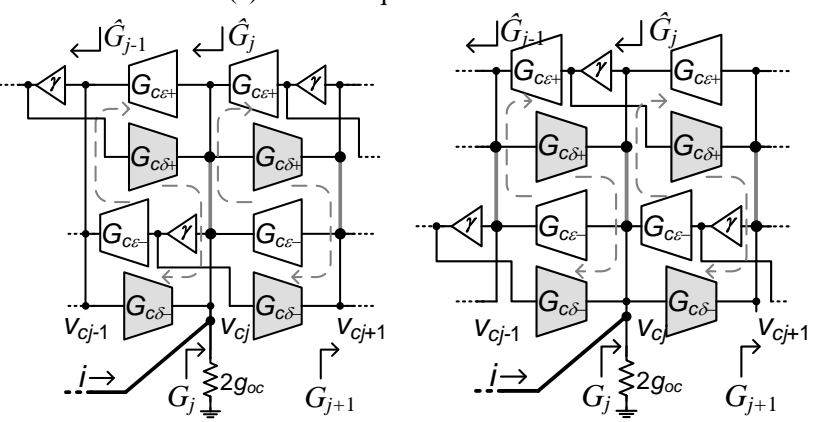

(b) Two possible feedback formations for loop gain $\left(L G_{j}\right)$ calculation

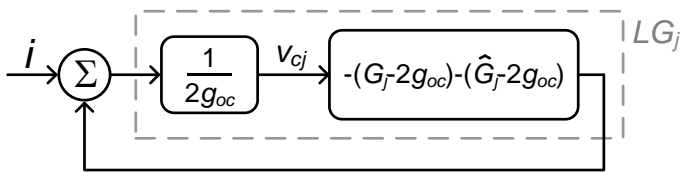

(c) Corresponding equivalent feedback system of (b)

Fig. 4. DC CM equivalent circuit of the SCC filter and loop gain calculation at node $j$ for $j=1,2, \ldots, N$.

It is also worth exploring the total grounded DC CM conductance, $G_{T j}$ at the $j^{\text {th }}$ node as an alternative to $L G_{j}$ for stability inspection. From the feedback system of Fig. 4(c), the loop gain, $L G_{j}$ can be directly linked with $G_{T j}$ by

$$
G_{T j}=i / V_{C j}=2 g_{o c}\left(1-L G_{j}\right)
$$

for $j=1,2, \ldots, N$. This total conductance $G_{T j}$ of Fig. 4(a)-(b) can also be seen from the circuit perspective, as the sum of two conductances $G_{j}$ and $\hat{G}_{j}$ subtracted by the excess (common) output conductance $2 g_{o c}$, i.e.

$$
G_{T j}=G_{j}+\hat{G}_{j}-2 g_{o c}
$$

for $j=1,2, \ldots, N$. With the $L G_{j}$ expression in (4), the conductance $G_{T j}$ relations in (5) and (6) are directly related. The filter is DC stable if this total conductance is positive, i.e., $G_{T j}>0$, which is the same condition as the stable feedback loop condition of $L G_{j}<1$ and this can also be seen from (5). Thus, the condition " $L G_{j}<1$ " or " $G_{T_{j}}>0$ " are equivalent for stability inspection. In the following discussion, only $G_{T j}$ will be used for stability assessment. The nodal conductance $G_{j}$ and $\hat{G}_{j}$ from (1) and (2) can be summarized in Table I with $\gamma=$ +1 for the conventional $G_{m}-C$ filter structure of Fig. 1 and with $\gamma=-1$ for the SCC $G_{m}-C$ filter structure of Fig. 3. Note also that if $\varepsilon=\delta= \pm 1$ for $\gamma=-1$, the SCC structure simply converges to the basic SCC $G_{m}-C$ filter structures in Fig. 2.

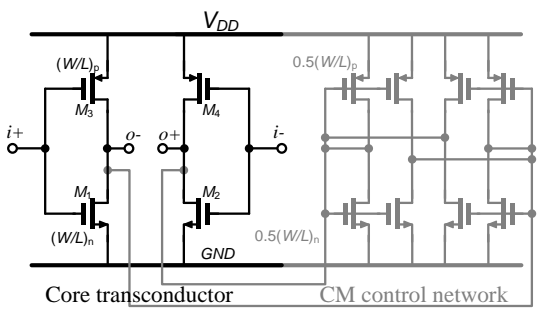

Fig. 5. A complete Nauta's inverter transconductor with a common-mode control network (in gray) [26]-[28]

TABLE I CM nodal conductances $G_{j}$ and $\hat{G}_{j}$ used for $G_{T j}$ calculation, $G_{T j}=G_{j}+\hat{G}_{j}-2 g_{o c}$ for $j=1,2, \ldots, N$

\begin{tabular}{|l|l|}
\hline $\begin{array}{l}\gamma=+1 \\
\text { (conventional) }\end{array}$ & $G_{j}=2 g_{o c}-\frac{G_{c}^{2}}{G_{j+1}}$ for $j=1,2, \ldots, N-1$ \\
\hline $\begin{array}{l}\gamma=-1 \\
\text { (SCC) }\end{array}$ & $G_{j}=2 g_{o c}-\frac{G_{c}^{2}}{\hat{G}_{j-1}}$ for $j=2,3, \ldots, N$ \\
& $\hat{G}_{j}=2 g_{o c}+\frac{\varepsilon \delta G_{c}^{2}}{\hat{G}_{j-1}}$ for $j=1,2, \ldots, N-1$ \\
\hline
\end{tabular}

$\hat{G}_{1}=G_{N}=2 g_{o c}+G_{c}$. If $\varepsilon=\delta=+1(-1)$, the SCC becomes the even- $($ odd - ) type basic SCC.

Since the pseudo-differential inverter-based transconductor $\left(M_{1}-M_{4}\right.$ in Fig. 5) will be the transconductor choice for the implementation and verification of the SCC filters in Sections II and III, it is instructive to use the CM analysis to examine DC stability in the conventional $G_{m^{-}} C$ filters of Fig. 1 based on such a transconductor. This complementary inverter-based transconductor is a core part of the complete Nauta's transconductor [26]-[28] in Fig. 5 where a CM control network is also included. By using Table $\mathrm{I}$, under the condition $\gamma=+1$ (" $\gamma$ " amplifiers associated with $G_{\varepsilon^{-}}$and $G_{\delta}$ in Fig. 4 become redundant), $\varepsilon=\delta=+1$ and $g_{o c} \approx 0$ as no CM control network is added and the transconductor ideally possesses a zero output conductance, with $N=$ odd this renders $\hat{G}_{j}=G_{j} \approx-G_{c}$ and $+G_{c}$ for $e v e n^{\text {th }}$ and $o d d^{\text {th }}$ nodes respectively. This consequently produces $G_{T j}=-2 G_{c}$ (even $^{\text {th }}$ nodes) and $+2 G_{c}$ (odd ${ }^{\text {th }}$ nodes) indicating an unstable filter. It is also important to note that for $\gamma=+1$ and $g_{o c} \approx 0$, even a perfect CM-rejection transconductor (i.e., $G_{c}=0$ ) could only give $G_{T j}$ $=0$ where $\mathrm{DC}$ stability cannot be guaranteed. The conventional filter can become stable by adopting the CM control network of the Nauta's transconductor (Fig. 5), which makes $g_{o c}=G_{c}$. Note that the Nauta transconductor's core possesses the same value of DM and CM transconductances, i.e., $G=G_{c}$ while its $\mathrm{CM}$ control network provides a zero DM transconductance with a $\mathrm{CM}$ transconductance of $G_{c}$. To simplify the analysis description, we firstly choose to omit the $\mathrm{CM}$ control network at the first and last nodes, i.e., by setting $g_{o c}=0$ for $j=1$ and $N$, while still keeping $g_{o c}=G_{c}$ for $j=2,3$, $\ldots, N-1$. Since $\hat{G}_{1}=G_{N} \approx+G_{c}$, the first row of Table I gives $\hat{G}_{j}$ $=G_{j} \approx+G_{c}$ for $j=2,3 \ldots, N-1$, and $G_{1}=\hat{G}_{N}=-G_{c}$ and $G_{T j}=0$ for all $j=1,2 \ldots, N$. This indicates that the above $g_{o c}$ setting yields a borderline condition between DC stable and unstable 
filter. By also setting $g_{o c}=G_{c}$ for $j=1$ and $N$ as employed in the original Nauta's $G_{m}-C$ filter [7], [26-29], the DC stability can thus be guaranteed. Although this DC stabilization technique has been demonstrated to be very effective, especially under a very low-voltage supply, it is at the expense of high power and large silicon area.

In case of the SCC filter with no extra CM control circuitry in Fig. 3(a) with $\gamma=-1$, applying the second row of Table I to (6) renders $G_{T j}=2 g_{o c}+\varepsilon \delta G_{c}^{2}\left(G_{j+1}^{-1}+\hat{G}_{j-1}^{-1}\right)$ for $j=2, \ldots, N-1$ where $G_{T 1}=G_{1}+G_{c}$ and $G_{T N}=\hat{G}_{N}+G_{c}$. Under a typical scenario where $g_{o c} \approx 0$, this simplifies $G_{j}$ and $\hat{G}_{j}$ in Table I to $G_{j} \approx+\varepsilon \delta G_{c}^{2} G_{j+1}^{-1}$ for $j=1,2 \ldots, N-1$ and $\hat{G}_{j} \approx+\varepsilon \delta G_{c}^{2} \hat{G}_{j-1}^{-1}$ for $j$ $=2,3 \ldots, N$. Since $\hat{G}_{1}=G_{N} \approx+G_{c}$, this further yields $G_{j}$ and $\hat{G}_{j}$ with $N=$ odd, to $G_{j}=\hat{G}_{j} \approx+\varepsilon \delta G_{c}$ with $G_{T j} \approx+2 \varepsilon \delta G_{c}$ for $j=$ even and $G_{j}=\hat{G}_{j} \approx+G_{c}$ with $G_{T j} \approx+2 G_{c}$ for $j=$ odd. However if $N=$ even, $G_{j}=\hat{G}_{k} \approx+\varepsilon \delta G_{c}$ for $j=$ odd, $k=$ even and $G_{j}=\hat{G}_{k} \approx+G_{c}$ for $j=$ even, $k=$ odd and this simply renders $G_{T j} \approx(1+\varepsilon \delta) G_{c}$ for all $j$. Therefore as long as $\varepsilon$ and $\delta$ are non zero and of the same polarity, i.e., $0<(\varepsilon, \delta) \leq+1$ or $-1 \leq(\varepsilon, \delta)$ $<0$, all of the conductances $G_{j}, \hat{G}_{j}$ and $G_{T j}$ are positive ensuring that the SCC filter is always stable. As previously noted with $\varepsilon=\delta=+1$ or -1 , the SCC filters simply turn into the basic SCC filters without any CM rejection as in Fig. 2(a). Moreover, with the condition $g_{o c} \approx 0$, these basic SCC filters possess $G_{1}=\hat{G}_{N} \approx+G_{c}, G_{j}=+G_{c}$ for $j=2, \ldots, N$, and $\hat{G}_{j}=+G_{c}$ for $j=1, \ldots, N-1$ resulting in $G_{T j} \approx+2 G_{c}$ for all $j$ which is higher than those from the SCC filters at $j=$ even implying a more stable condition offered by the basic SCC filters. Conclusively, the inclusion of the inverting amplifiers in the SCC filters fundamentally makes all of the total node conductances positive. As a result, these SCC filter structures are inherently DC stable.

When $\varepsilon, \delta$ are close to zero with $N=$ odd, then $G_{T j}$ also approaches zero for $j=$ even. This simply pushes the filter very close to the unstable region. Although a CM control network can be included (i.e. to set $g_{o c} \gg 0$ ) to guarantee stability, this may inevitably incur significant cost to power consumption. A more practical approach is to keep the condition $0<(\varepsilon, \delta)<+1$ or $-1<(\varepsilon, \delta)<0$ and find a suitable trade-off between the stability and $\mathrm{CM}$ rejection for a particular filter implementation. This will be discussed in the next subsection.

\section{DC CM Gain Analysis and Design Trade-off}

The DC CM voltage at each stage, $V_{C j}$ of the SCC Filter in Fig. 4(a) for $j=2,3 \ldots, N$ can be expressed for $N=$ odd (even) as

$$
\begin{aligned}
& V_{C j}=V_{C j-1} \frac{G_{c}}{2}(\gamma(1-\delta)+(1+\delta)) G_{j}^{-1}, j=\text { even (odd) } \\
& V_{C j}=V_{C j-1} \frac{G_{c}}{2}((1-\delta)+\gamma(1+\delta)) G_{j}^{-1}, j=\text { odd (even). }
\end{aligned}
$$

If a perfect inverting amplifier is assumed, i.e., $\gamma=-1$, the magnitude of these DC CM voltages become

$$
\left|V_{C j}\right|=\left|V_{C j-1}\right| \delta G_{c} G_{j}^{-1} .
$$

for $j=2,3 \ldots, N$ and $\left|V_{C 1}\right|=\left|V_{i C}\right| G_{c i} G_{T 1}^{-1}$. Following the $G_{j}$ and $\hat{G}_{j}$ values of the SCC filter with $g_{o c} \approx 0$ in Section II.C, we have $G_{T 1}=2 G_{c}$ for $N=$ odd (only an odd $d^{\text {th }}$-order filter is considered due to its popularity over its $e^{e^{\text {th }}}{ }^{\text {th }}$-order counterpart). If $G_{c i}=G_{c}$, the DC CM voltage at first node $V_{c 1}$ from the DC CM input voltage $V_{i c}$ then becomes $V_{c 1}=V_{i c} / 2$ for $N=$ odd. Therefore the $N^{\text {th }}$-order filter's CM DC gain, $A_{c m}$ can be found by using $(7 \mathrm{c})$ recursively as

$$
A_{c m}=\frac{V_{o c}}{V_{i c}}=\frac{1}{2}\left(\frac{\delta}{\varepsilon}\right)^{\left(\frac{N-1}{2}\right)} \quad \text { for } N=\text { odd }, N \geq 3
$$

The above equation (8) indicates that a small $\delta / \varepsilon$ ratio is required for a good CM rejection. As already shown in Section II.C, with $N=$ odd, the product of $\varepsilon \cdot \delta$ should be sufficiently large to obtain a positively large $G_{T j}\left(\approx+2 \varepsilon \delta G_{c}\right)$ at $j=$ even, for a high degree of DC stability. As a consequence, there is a design trade-off for the $o d d^{\text {th }}$-order SCC filter between the stability (i.e., $G_{T j}$ values) and the DC CM gain $\left(A_{c m}\right)$, by balancing $\varepsilon$ and $\delta$ to provide a stable filter with $\mathrm{CM}$ rejection that meets the required specifications.

\section{E. Overhead Power Comparison: Inverter-based Pseudo- Differential Transconductor Case}

In order to quantify the power saving offered by the SCC structures, a comparison between the overhead power consumption required for $\mathrm{CM}$ stabilization in an $N^{\text {th }}$-order lowpass $G_{m}-C$ filter using the proposed and conventional structures is given. Due to its class- $\mathrm{AB}$ operation and versatility for a very low supply voltage, the complementary inverter-based pseudo-differential pair is chosen as the core transconductor $G$ of the filters. In the conventional structure, each of these individual transconductors is equipped with the CM control network which resembles a complete Nauta's transconductor [7], [26]-[30]. In the SCC structures of Fig. 2(a) and Fig. 3(a), the same core transconductor is also employed to implement the inverting amplifiers.

Since the relation between the quiescent supply current and transconductance is approximately linear in the inverter-based transconductor, the overhead power comparison can be directly related to the added transconductance for CM stabilization and control. In the conventional $N^{\text {th }}$-order lowpass $G_{m}-C$ filter using the Nauta's type CM network, it is straightforward to show that the added transconductance is $G_{C o h}=(2 N+1) G$. In the SCC $N^{\text {th }}$-order filter counterparts, the added transconductance is from the inverting amplifier, which requires $2 \alpha$ basic units $(G$ or $G / 2)$ of the pseudo-differential transconductors for each implementation, e.g. $\alpha=1$ as in Fig. 2(a) and Fig. 3(a). For the basic even-type SCC filter structure similar to the left one in Fig. 2(a), its $G_{C o h}$ is equal to $\alpha N \cdot G$ and $\alpha(N-1) G$ for $N=$ even number and odd number, respectively. While for the basic odd-type SCC structure (the right one in Fig. 2(a)), the $G_{C o h}$ becomes $\alpha N \cdot G$ and $\alpha(N+1) G$ 


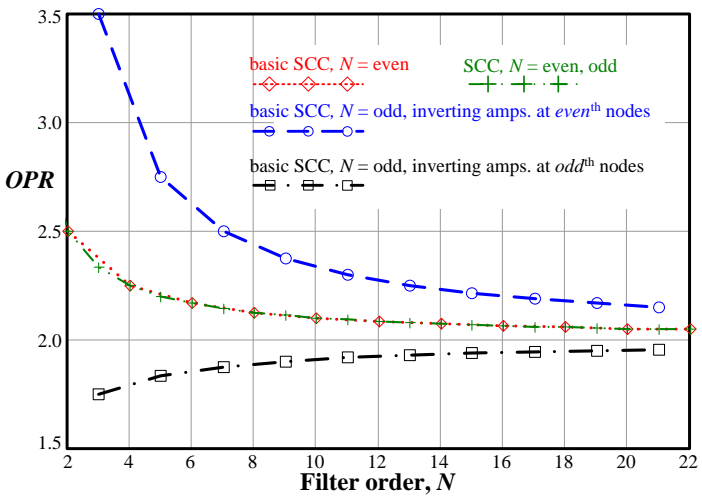

Fig. 6. Overhead power ratio $(O P R)$ between the conventional and the proposed self-stabilized for the $N^{\text {th }}$-order elliptic lowpass filter with $\alpha=1$.

respectively for $N=$ even and odd. Whereas for the SCC structure as in Fig. 3(a), the $G_{C o h}$ is $\alpha N \cdot G$ for even and odd $N$. Therefore, the overhead power ratio, $O P R$, between the conventional and the basic SCC or the SCC filters is given by

$$
O P R=(2 N+1) /(\alpha(N+c)),
$$

where $c=0$ for the SCC structure with $N=$ even and for both the basic even- and odd-type SCC structures. But for the basic SCC structures with $N=$ odd, $c$ becomes -1 and +1 for the even- and odd-type configurations, respectively. Specifically for a $5^{\text {th }}$-order lowpass filter and $\alpha=1$, equation (9) gives $O P R=11 / 4(11 / 6)=2.75(1.83)$ for the even- $($ odd -$)$ type basic SCC filter in Fig. 2(a) and $O P R=11 / 5=2.2$ for the SCC filter in Fig. 3(a). The equation also suggests that for a very high order filter, $N \gg 1$, the $O P R$ converges to 2 which is equivalent to $50 \%$ overhead-power saving. This implies that, by using the inverter-based pseudo-differential circuit as the core transconductor, the conventional filter structure essentially requires twice as much overhead power as that required in the SCC structures for $\mathrm{CM}$ stabilization. Plot of the $O P R^{\prime}$ s versus $N$ using (9) is displayed in Fig. 6.

\section{LOW-Power Differential CoMPlex GM-C FiLter USING SELF-CM-CONTROL STRUCTURE}

\section{A. Self-CM-Controlled (SCC) Differential Complex $G_{m}-C$ Filter with Common-Mode Rejection}

In the conventional complexification technique for differential $G_{m}-C$ filters, appropriate pairs of crossing transconductors are introduced between $I$ and $Q$ lowpass filter (LPF) sections. These include one crossing transconductor pair, $G_{x j} \mathrm{~s}\left(=\omega_{0} C_{j}\right)$, for each grounded capacitor $C_{i}$ (as also shown in Fig. 7(a)), and four crossing transconductor pairs, $G_{x i j} \mathrm{~s}\left(=\omega_{0} C_{i j}\right)$, for each floating capacitor $C_{i j}$, where $\omega_{0}$ is the center radian frequency of the complex filter [5], [7], [29-30], [40]. Since these $G_{x i}$ and $G_{x i j} \mathrm{~s}$ are connected in a head-to-tail fashion similar to those core transconductors, $G$ s, inside the $I$ and $Q$ LPFs, the complexification structure inevitably introduces more $\mathrm{CM}$ positive feedback loops. In order to suppress the resulting CM positive feedback effect and hence stabilize the complex filter, each of these $G_{x i} \mathrm{~s}$ and $G_{x i j} \mathrm{~s}$ must require a $\mathrm{CM}$ control network in addition to those already needed in the $I$ and $Q$ LPF sections. This, as a result, significantly increases power and silicon area.
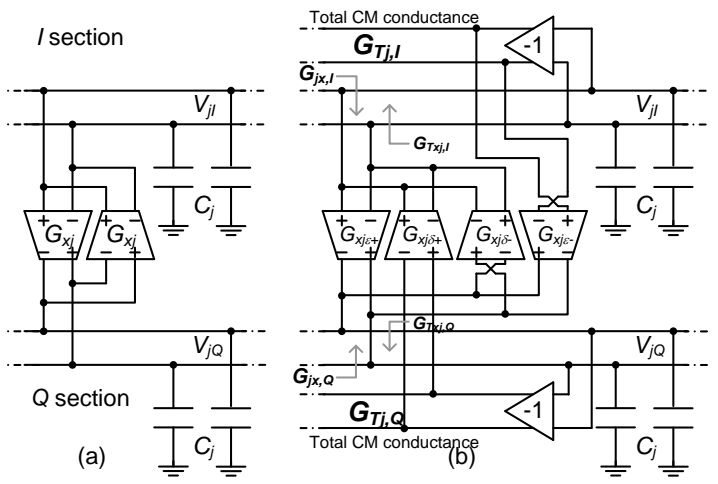

Fig. 7. Implementation for complexification with $G_{x j}$ : (a) conventional (b) employing the SCC feedback structure from Fig. 3(a) with $G_{x j \hbar \pm}=$ $\left(1 \pm \varepsilon_{x}\right) G_{x j} / 2, G_{x j \delta \pm}=\left(1 \pm \delta_{x}\right) G_{x j} / 2$.

Fig. 7(b) shows a differential implementation example for the crossing $G_{x j}$ at any node $j$ with grounded $C_{j}$ where the $I$ and $Q$ sections utilize the proposed SCC filter structure from Fig. 3(a) and each $G_{x j}$ in Fig. 7(a) has been split into a pair of $G_{x j \varepsilon \pm}$ and $G_{x j \delta \pm}$ as similar to the SCC structure in Fig. 3(a) with $G_{x j \xi \pm}=\left(1 \pm \varepsilon_{x}\right) G_{x j} / 2, G_{x j \delta \pm}=\left(1 \pm \delta_{x}\right) G_{x j} / 2$. Co-operation with two half-sized inverting amplifiers already existed in the $I$ and $Q$ sections gives two necessary differential negative feedback loops for complexification. Since each of these loops essentially involves one inverting amplifier, this thus prevents $\mathrm{CM}$ positive feedback loops inevitably introduced in the conventional differential complex filter (Fig. 7(a)). Regarding to a CM signal, the second row of Table I can also be applied to Fig. 7(b) for calculating the CM conductance $G_{j x, I(Q)}$ looking into the complexification $G_{x j \varepsilon \pm},\left(G_{x j \delta \pm}\right)$ network from the $I(Q)$ section at $j^{\text {th }}$ node as influenced by the $G_{T x j, Q(I)}$ from the opposite $Q(I)$ section, i.e., $G_{j x, I(Q)} \approx+\varepsilon_{x} \delta_{x} G_{x j c}^{2} G_{T x j, Q(I)}^{-1}$. The offsets $\varepsilon_{x}$ and $\delta_{x}$ can thus be assigned to modify the resultant total CM nodal conductance, $G_{T j}$ on both $I$ and $Q$ sections namely $G_{T j, I}$ and $G_{T j, Q}$. This complexification technique for $G_{x j}$ 's can be directly applied for $G_{x i j}$ 's (split into $G_{x i j \xi \pm}$ and $G_{x i j \delta \pm}$ with $\left.G_{x i j \delta \pm}=\left(1 \pm \varepsilon_{x}\right) G_{x i j} / 2, G_{x i j \delta \pm}=\left(1 \pm \delta_{x}\right) G_{x i j} / 2\right)$ and this eventually renders a fully-functional low-power $5^{\text {th }}$-order SCC complex filter as shown in Fig. 8 (illustrated with $\varepsilon_{x}, \delta_{x}=0$ ).

The ideal inverting amplifiers with perfect matching among $G_{x j} / 2$ 's and $G_{x i j} / 2$ 's $\left(\varepsilon_{x}, \delta_{x}=0\right)$ would result in a complete CM signal cancellation from $I$ section to $Q$ section and vice versa, i.e. $G_{x j} / 2$ 's and $G_{x i j} / 2$ 's become open circuit for $\mathrm{CM}$ signal. This would basically isolate $I$ and $Q$ sections from each other yielding the complex filter's CM response and stability characteristic that closely converges to those of the two separate stable SCC LPFs. Thus, it follows that the complex filter of Fig. 8 requires neither extra CM control network nor additional inverting amplifiers, apart from the existing ones in the $I$ and $Q$ LPF sections. As a consequence, the proposed SCC filter structure of Fig. 3(a) is even more efficient in terms of power and area consumptions when utilized for complex filter implementations.

An impact by the inverting amplifier's finite bandwidth on the imbalances of the $I$ and $Q$ signals from the required quadrature phase shift may be investigated by considering only the even nodes for simplicity. A differential-mode voltage at the node $j$ (for $j=2,4 \ldots$ ) of the combined $I-Q$ sections can be given by 
1

2

3

4

5

6

7

8

9

$$
\begin{aligned}
V_{j, I}= & {\left[\left(-V_{j-1, I}\right)\left(-G_{\delta+}+\gamma G_{\delta-}\right)+V_{j+1, I}\left(-G_{\varepsilon+}+\gamma G_{\varepsilon-}\right)\right] / s\left(C_{j} / 2\right)+\ldots } \\
& +\left(-G_{x j \delta-}+\gamma G_{x j \delta+}\right)\left(+V_{j, Q}\right) / s\left(C_{j} / 2\right) \\
V_{j, Q}= & {\left[\left(-V_{j-1, Q}\right)\left(-G_{\delta+}+\gamma G_{\delta-}\right)+V_{j+1, Q}\left(-G_{\varepsilon+}+\gamma G_{\varepsilon-}\right)\right] / s\left(C_{j} / 2\right)+\ldots } \\
& +\left(-G_{x j \varepsilon+}+\gamma G_{x j \varepsilon-}\right)\left(-V_{j}^{I}\right) / s\left(C_{j} / 2\right) .
\end{aligned}
$$

The above equations suggest that the perfect quadrature phase relations between the $I$ and $Q$ voltages can be realized by setting $G_{x j \delta+(-)}=G_{x j \delta_{-(+)}}$with $\varepsilon_{x}=-\delta_{x}$ (including $\varepsilon_{x}, \delta_{x}=0$ as in Fig. 8) because the factors $\gamma$ associated with the inverting amplifier's response are perfectly balanced within the $I$ and $Q$ sections. Since the same conclusion applies for the odd nodes, it can be deduced that the complex filter's balanced characteristic between $I$ and $Q$ sections can be maintained regardless of the inclusion of the inverting amplifiers in the differential SCC complex filter. The crossing $G_{x j \varepsilon \pm}, G_{x j \delta \pm}$ network renders a negative CM conductance $G_{j x, I(Q)}$ for $\varepsilon_{x}=$ $-\delta_{x}$ (non-zero values). A similar recursive calculation on the second row of Table I suggests that if this negative $G_{j x, I(Q)}$ is applied to the odd nodes only, it would effectively modify the original $G_{T j}$ 's of the simple SCC complex filter (with $\varepsilon_{x}=\delta_{x}=$
0 ) such that the $G_{T j}$ 's at $j=o d d$ (even) is decreased (increased) from $+2 G_{c}\left(+2 \varepsilon \delta G_{c}\right)$. This in turn helps reduce the difference of the resultant $G_{T j}$ within the SCC complex filter and bring the quiescent CM DC voltage levels (including the bias voltages) among all the nodes closer to each other. However, to ensure the SCC complex filter's stability with this $\varepsilon_{x}=-\delta_{x}$ condition, the magnitude of $\varepsilon_{x} \cdot \delta_{x}$ has to be sufficiently small so that all the modified $G_{T j}$ 's remained positive.

\section{B. Overhead Power Consumption: An Inverter-Based Pseudo-Differential Transconductor Study Case}

By using the overhead power ratio $O P R$, the complex $G_{m^{-}}$ $C$ filters based on the conventional and SCC structures are compared in terms of the added power consumption necessary to achieve the CM DC stabilization. Since the complex filter with the elliptic characteristic is the main focus in this work, only the ratio analysis for an odd-order filter is given. In the conventional structure, it requires that the $\mathrm{CM}$ stabilization network of the Nauta's type be employed for all the core and the crossing transconductors $G$ 's, $G_{x i}$ 's, $G_{x i j}$ 's [7], [29-30]. By contrast, no additional circuit is required in the SCC filter, since the embedded inverting amplifiers readily provide $\mathrm{CM}$ negative feedbacks for both internal and cross-coupling loops.

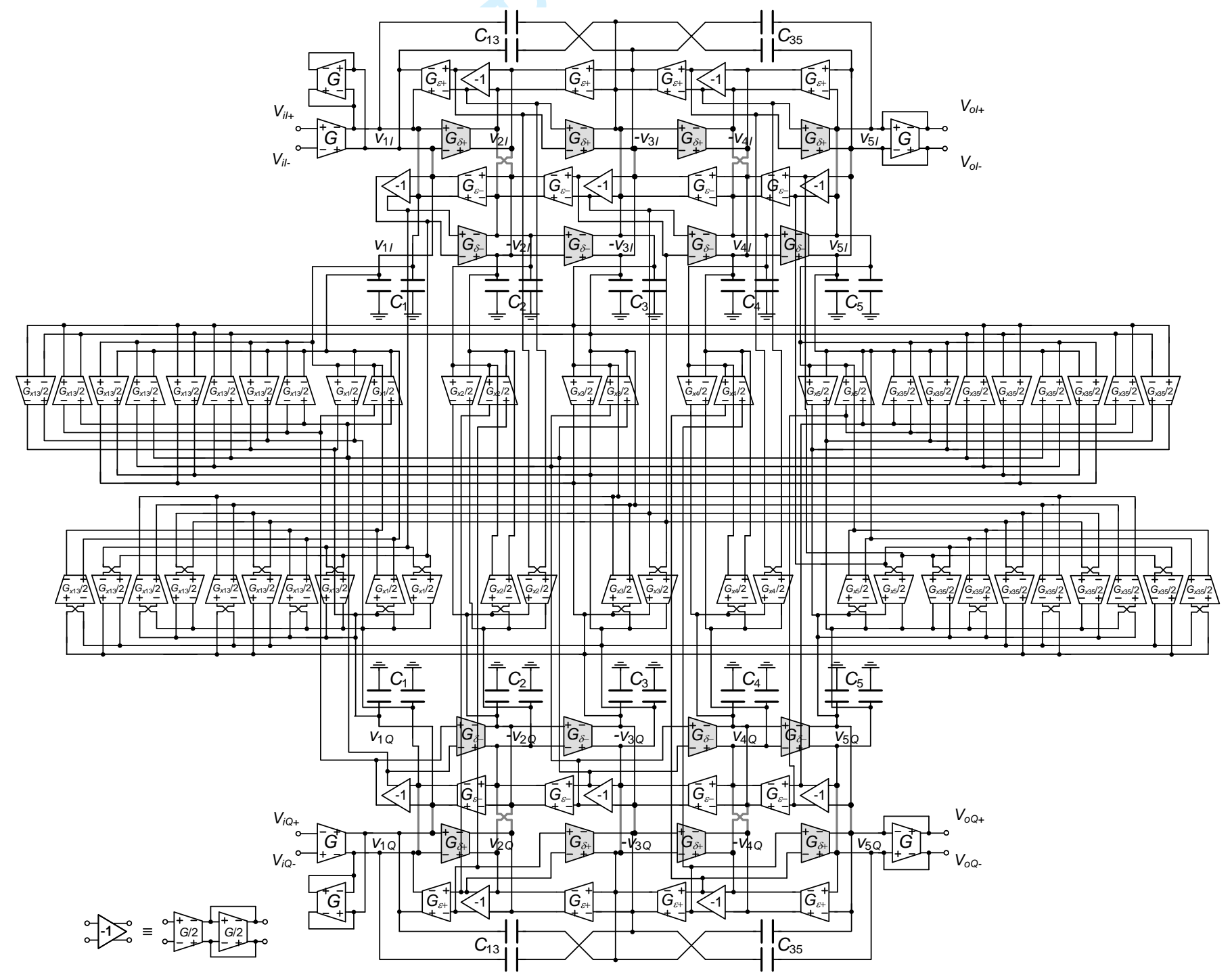

Fig. 8. Proposed low-power SCC $G_{m^{-}} C$ complex filter structure with CM rejection where $G_{\varepsilon \pm}=(1 \pm \varepsilon) G / 2, G \delta \pm(1 \pm \delta) G / 2, \alpha=1$ and $\varepsilon_{x}, \delta_{x}=0$. 


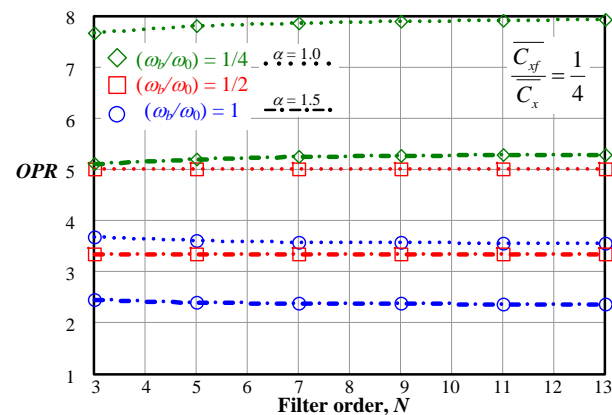

(a) $O P R v s N v s \omega_{b} / \omega_{0}\left(o d d^{\text {th }}\right.$ order only)

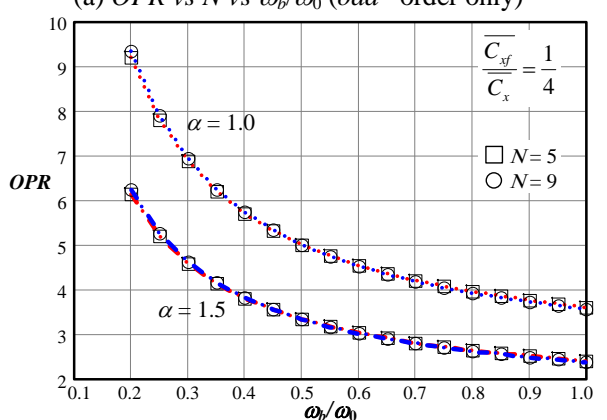

(b) $O P R$ vs $\left(\omega_{b} / \omega_{0}\right)$

Fig. 9. Overhead power ratio by the SCC $N^{\text {th }}$-order elliptic complex filter from (28) ( $N=$ order of the $I, Q$ lowpass sections) with $\alpha=1.0,1.5$.

Similar to the analysis in Section II.E, the overhead power comparison is determined by the added transconductance for CM stabilization. For complexification, since each of the $N$ grounded capacitors requires two crossing transconductors $G_{x i}$ and each of the $(N-1) / 2$ floating capacitors needs eight crossing transconductors $G_{x i j}$, the $O P R$ can be written as

$$
O P R=\left(2(2 N+1)+\left(\sum_{i=1}^{N} 2 \frac{G_{x i}}{G}+\sum_{j}^{(N-1) / 2} 8 \frac{G_{x(2 j-1)(2 j+1)}}{G}\right)\right) /(\alpha 2 N)
$$

with $G_{x i}=\omega_{0} C_{i}(i=1, \ldots, N), G_{x(2 j-1)(2 j+1)}=\omega_{0} C_{(2 j-1)(2 j+1)}(j=1$, $\ldots,(N-1) / 2)$. By putting the average values of $G_{x i}$ and $G_{x(2 j-}$ 1)(2j+1) as $\overline{G_{x}}$ and $\overline{G_{x f}}$, respectively, (11) is simplified to

$$
O P R=\left(2(2 N+1)+\left(2 N \frac{\overline{G_{x}}}{G}+\frac{(N-1)}{2} 8 \frac{\overline{G_{x f}}}{G}\right)\right) /(\alpha 2 N)
$$

To investigate how the $O P R$ is dependent on the complex filter's bandwidth $\left(2 \omega_{b}\right)$ and center frequency $\left(\omega_{0}\right)$, the $I / Q$ LPF section's bandwidth $\left(\omega_{b}\right)$ can be approximately related to the core transconductance, $G$, and the average grounded capacitance, $\bar{C}\left(=\overline{G_{x}} / \omega_{0}\right)$ by $\omega_{b} \cong G / \bar{C}$. Together with the average value of the floating capacitance $\overline{C_{x f}}\left(\cong \overline{G_{x f}} / \omega_{0}\right)$, the $O P R$ in (11) can alternatively be expressed as

$$
O P R \cong\left(2(2 N+1)+\left(\omega_{b} / \omega_{0}\right)^{-1}\left(2 N+\frac{(N-1)}{2} 8\left(\overline{C_{x f}} / \overline{C_{x}}\right)\right)\right) /(\alpha 2 N)
$$

For a special case of the elliptic complex filter with $\omega_{b} / \omega_{0}=$ $1 / 2$ and $\overline{C_{x f}} / \overline{C_{x}}=1 / 4$ as in the design in Section IV, both (12) and (13) yield $O P R=5 / \alpha$, e.g., the $O P R$ s are 5.0 and 3.3 for $\alpha$ $=1.0$ and 1.5 , respectively. Note that, the factor $\alpha$ plays a vital role in the filter's noise performances as will be analyzed in Section III.D. This specifically indicates that based on the inverter-based pseudo-differential transconductors, the

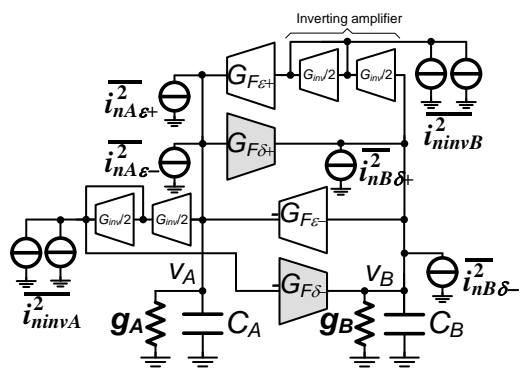

Fig. 10. Single-ended biquadratic structure for noise investigation as impacted by the inverting amplifier with $G_{F \varepsilon \pm}=(1 \pm \varepsilon) G_{F} / 2, G_{F \delta \pm}=(1 \pm \delta) G_{F} / 2$ and $G_{i n v}=\alpha_{F} G_{F}, \overline{i_{n i n v(A, B)}^{2}}=\alpha_{F} \overline{i_{n G f}^{2}} / 2$

conventional complex filter requires at least three times as much overhead power as that required in the SCC complex filter for CM stabilization. The significant power saving in the proposed complex filter simply stems from the fact that there is no overhead power required for those crossing transconductors $G_{x i}, G_{x i j}$.

Plots of the $O P R$ s using (13) are illustrated in Fig. 9 showing how $O P R$ is related to the filter's order $N$ (= odd) and the bandwidth/center frequency ratio $\omega_{b} / \omega_{0}$ for $\alpha=1.0$ and 1.5 . As evident from the plots, the $O P R$ is almost constant against the filter's order (Fig. 9(a)). Also for a high-order filter $(N \geq$ $5)$, the $O P R$ is inversely proportional to $\omega_{b} / \omega_{0}$ (Fig. 9(b)). This is mainly because of the relation $\omega_{b} \propto G$ where a larger $\omega_{b}$ results in a larger $G$, making the effect of no added power in $G_{x i}$ and $G_{x i j}$ in the SCC filter less significant.

\section{Effect of Inverting Amplifier on Noise Performance}

The impact of the inverting amplifiers on the noise characteristic of the SCC filter is analyzed using a secondorder circuit, which serves as the core structure of both higherorder and complex filters, as shown in Fig. 10 where $G_{F \varepsilon \pm}=$ $(1 \pm \varepsilon) G_{F} / 2, G_{F \delta \pm}=(1 \pm \delta) G_{F} / 2$ with $G_{F}=G$ for the lowpass sections' transconductors, and $G_{F}=G_{x i}$ and $G_{x i j}$ of the crossing transconductors. The inverting amplifier comprises a pair of pseudo-differential transconductors, with $G_{i n v}=\alpha_{F} G_{F}$. The conductor-capacitor networks, $\left(g_{A}+s C_{A}\right)$ and $\left(g_{B}+s C_{B}\right)$ have been included to model adjacent second-order stage loading. Also shown in Fig. 10 are noise sources, which model the thermal noise associated with the transconductors $G_{F \varepsilon \pm}, G_{F \delta \pm}$ and $G_{i n v} / 2$, where $\overline{i_{n A \varepsilon \pm}^{2}}=(1 \pm \varepsilon) \overline{i_{n G_{F}}^{2}} / 2$ and $\overline{i_{n B \delta \pm}^{2}}=(1 \pm \delta) \overline{i_{n G_{F}}^{2}} / 2$ with $\overline{i_{n G_{F}}^{2}}=\gamma_{G} k T G_{F}$ and $\overline{i_{n i n v(A, B)}^{2}}=\gamma_{G} k T G_{i n v} / 2=\alpha_{F} \overline{i_{n G_{F}}^{2}} / 2$. The conductors' noise may also be included but this is omitted for simplicity. The mean-square noise voltages $\overline{v_{n A}^{2}}$ and $\overline{v_{n B}^{2}}$ generated from these uncorrelated current noise sources were analyzed as summarized in Table II.

For the mean-square noise voltages due to $\overline{i_{n A}^{2}}, \overline{i_{n B}^{2}}$ in Table II, they are the same as the noise transfers in the second-order filter without the inverting amplifier. This thus indicates no difference between the noise characteristics of the SCC and conventional filters due to these sources. On the other hand, the mean-square noise voltages due to $\overline{i_{\operatorname{ninv}(A, B)}^{2}}\left(=\alpha_{F} \overline{i_{n G_{F}}^{2}} / 2\right)$ indicate that the noise contribution from the inverting amplifier's transconductor is dependent on the factor $\alpha_{F}$ (= 
TABLE II NOISE TRANSFER FROM VARIOUS NOISE SOURCES IN FIG. 10

\begin{tabular}{|c|c|}
\hline$\overline{i_{n A}^{2}} \rightarrow \overline{v_{n A}^{2}}, \overline{v_{n B}^{2}}$ & $\overline{v_{n A}^{2}}=\overline{i_{n A}^{2}} \frac{\left|g_{B}+j \omega C_{B}\right|^{2}}{D(j \omega)}, \overline{v_{n B}^{2}}=\overline{i_{n A}^{2}} \frac{\left|G_{F}\right|^{2}}{D(j \omega)}$ \\
\hline$\overline{i_{n B}^{2}} \rightarrow \overline{v_{n A}^{2}}, \overline{v_{n B}^{2}}$ & $\overline{v_{n A}^{2}}=\overline{i_{n B}^{2}} \frac{\left|G_{F}\right|^{2}}{D(j \omega)}, \overline{v_{n B}^{2}}=\overline{i_{n B}^{2}} \frac{\left|g_{A}+j \omega C_{A}\right|^{2}}{D(j \omega)}$ \\
\hline$\overline{i_{\text {ninvA }}^{2}} \rightarrow \overline{v_{n A}^{2}}$ & $\overline{v_{n A}^{2}}=\overline{i_{n i n v A}^{2}} \frac{(1-\delta)^{2}\left|g_{A}+j \omega C_{A}\right|^{2}}{\alpha_{F}^{2} D(j \omega)}=\overline{i_{n B \delta-}^{2}} \frac{(1-\delta)\left|g_{A}+j \omega C_{A}\right|^{2}}{\alpha_{F} D(j \omega)}$ \\
\hline$\overline{i_{n i n v A}^{2}} \rightarrow \overline{v_{n B}^{2}}$ & $\overline{v_{n B}^{2}}=\overline{i_{n i n v A}^{2}} \frac{(1-\delta)^{2} G_{F}^{2}}{\alpha_{F}^{2} D(j \omega)}=\overline{i_{n B \delta-}^{2}} \frac{(1-\delta) G_{F}^{2}}{\alpha_{F} D(j \omega)}$ \\
\hline$\overline{i_{n i n v B}^{2}} \rightarrow \overline{v_{n A}^{2}}$ & $\overline{v_{n A}^{2}}=\overline{i_{n i n v B}^{2}} \frac{(1+\varepsilon)^{2}\left|g_{A}+j \omega C_{A}\right|^{2}}{\alpha_{F}^{2} D(j \omega)}=\overline{i_{n A \varepsilon+}^{2}} \frac{(1+\varepsilon)\left|g_{A}+j \omega C_{A}\right|^{2}}{\alpha_{F} D(j \omega)}$ \\
\hline$\overline{i_{\text {ninvB }}^{2}} \rightarrow \overline{v_{n B}^{2}}$ & $\overline{v_{n B}^{2}}=\overline{i_{n i n v B}^{2}} \frac{(1+\varepsilon)^{2} G_{F}^{2}}{\alpha_{F}^{2} D(j \omega)}=\overline{i_{n A \varepsilon+}^{2}} \frac{(1+\varepsilon) G_{F}^{2}}{\alpha_{F} D(j \omega)}$ \\
\hline
\end{tabular}

$\left.G_{i n v} / G_{F}\right)$. In case of a lowpass filter design using the SCC structure with small $\varepsilon, \delta$ and $\alpha_{F}=1$, i.e., $G_{i n v}=G_{F}=G$, the mean-square noise transfers due to $\overline{i_{\operatorname{minv}(A, B)}^{2}}$ is similar to those due to $\overline{i_{n G_{F}}^{2}}$ 's. However, because the total transconductance is smaller, the SCC filter structure therefore exhibits less total noise as compared to the conventional filter with $\mathrm{CM}$ control networks. Specifically at low frequencies, the SCC lowpass filter can provide total thermal noise reduction by a similar degree to the power saving factor as discussed in Section II.E.

In case of a complex filter design, the crossing transconductors $G_{x i}$ 's and $G_{x i j}$ 's can be treated as $G_{F}$ in the second-order circuit of Fig. 10. The values of $G_{x i}$ 's are typically larger than $G$ in the lowpass $I, Q$ sections depending on the $\omega_{0} / \omega_{b}$ ratio. As a consequence, with $G_{i n v}=G$, the factor $\alpha_{F}\left(=G_{i n v} / G_{x i}=G / G_{x i}\right)$ could be much less than unity. As indicated in Table II, the noise contribution from $\overline{i_{\text {ninv }(A, B)}^{2}}$ is essentially increased by the factor $1 / \alpha_{F}^{2}$. This adverse effect must be taken into account when designing a SCC complex filter. As adopted in the prototype filter in Section IV, a general design guideline is to select the transconductance $G_{i n v}>G$ so that the factor $\alpha_{F}$ is increased and the SCC complex filter exhibits an overall noise performance comparable to that of its conventional counterpart, while still enjoying a significant amount of power saving.

\section{Effect of Inverting Amplifier's Parasitic Capacitance on} Frequency Response

The non-ideal effect due to the inverting amplifier's parasitic capacitor $C_{i n v}$ on the filter's frequency response can be investigated from the single-ended $2^{\text {nd }}$-order SCC circuit as shown in Fig. 11. The impedance looking into node $X, Z_{X}$ can be expressed as

$$
Z_{X}=\frac{s C_{L}\left(G_{F} / 2\right)^{-2}}{\left[(1+\delta)+\frac{(1-\delta)}{1+s C_{\text {inv }} / G_{\text {iv }}}\right]\left[(1-\varepsilon)+\frac{(1+\varepsilon)}{1+s C_{\text {inv }} / G_{\text {inv }}}\right]} .
$$

To provide insight and, at the same time, simplify the analysis, this SCC structure can be reduced to a simple even- or oddtype basic SCC structure with $\varepsilon=\delta=+1$ or -1 , respectively where the second-order circuit is transformed into an

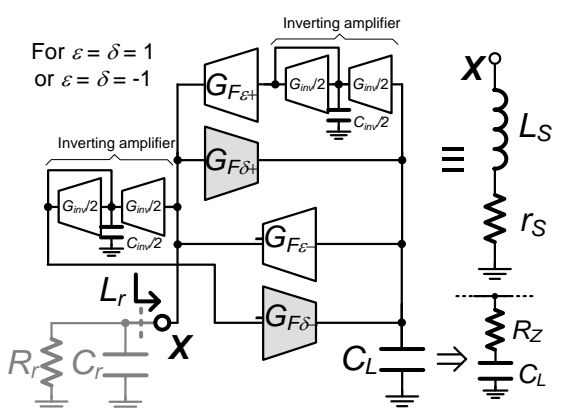

Fig. 11. DM single-ended biquad for study on the impact of the inverting amplifier's $C_{i n v}$ on frequency response with $G_{F \varepsilon \pm}=(1 \pm \varepsilon) G_{F} / 2, G_{F \delta \pm}=$ $(1 \pm \delta) G_{F} / 2$. Resistor $R_{Z}$ in series with $C_{L}$ is for compensation.

equivalent parallel $R L C$ circuit as also provided in Fig. 11. The equivalent series inductor $L_{S}$ and resistor $r_{S}$ are given by

$$
L_{S}=C_{L} / G_{F}^{2}, R_{S}=-\omega^{2}\left(C_{L} / G_{F}^{2}\right)\left(C_{i n v} / G_{i n v}\right) .
$$

As indicated by (14b), the capacitance $C_{i n v}$ has no impact on the inductance $L_{S}$. On the contrary, it gives rise to $r_{S}$, which is proportional to $C_{i n v}$ and is of a frequency-dependent negative resistance (FDNR) type. This thus results in a higher quality factor of the parallel $R L C$ circuit than the designed value, and the effect is more pronounced at higher frequencies. Another non-ideal effect is on the resonant frequency $\omega_{r}$, defined as the frequency that yields zero imaginary part in the admittance of the $R L C$ circuit. It is given by

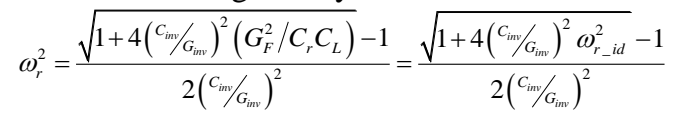

when $C_{i n v}$ approaches zero, by using the approximation $(1+x)^{\frac{1}{2}} \approx 1+(x / 2)$, we have the ideal resonant frequency at $\omega_{r-i d}=G_{F} / \sqrt{C_{r} C_{L}}$. By rearranging (15) as follows:

$$
\omega_{r_{-} i d}^{2} / \omega_{r}^{2}=1+\left(C_{i m p} / G_{i v i v}\right)^{2} \omega_{r}^{2},
$$

and since all the variables are positive, it can be deduced that $\omega_{r-i d}>\omega_{r}$. Thus, the effect of $C_{i n v}$ results in a lower resonant frequency than the designed value. Both the increase in the quality factor and the decrease in the resonant frequency due to $C_{i n v}$ invariably change the frequency response of the biquad circuit, and hence distort the overall frequency response of the filter. Therefore the parasitic $C_{i n v}$ must be kept sufficiently small to maintain the desired response, unless a suitable compensation technique is introduced as described next.

\section{E. Series-Resistor Technique for Parasitic Pole Cancellation in SCC Filter}

As widely employed in integrated continuous-time filters, and extensively explored in [41], the technique employing a passive resistor $R_{Z}$ in series with the capacitor $C_{L}$ can be used to compensate for the effect of $C_{i n v}$ from the inverting amplifier associated with $C_{L}$ in the $2^{\text {nd }}$-order SCC filter of Fig. 11. The resistor $R_{Z}$ effectively introduces a zero at $-1 / R_{Z} C_{L}$ at the inverting amplifier's input voltage. This helps cancel out the parasitic pole at at $-G_{i n v} / C_{i n v}$ at its output voltage, thereby enabling the associated $G_{F \varepsilon+}\left(=(1+\varepsilon) G_{F}\right)$ to produce the output current with no magnitude or phase distortion as compared to the ideal case when $C_{i n v}=0$.

This simple series-resistor technique can be extended to compensate for the effect of $C_{i n v}$ from any inverting amplifier in a general SCC filter structure. Similar to the case of Fig. 11, 


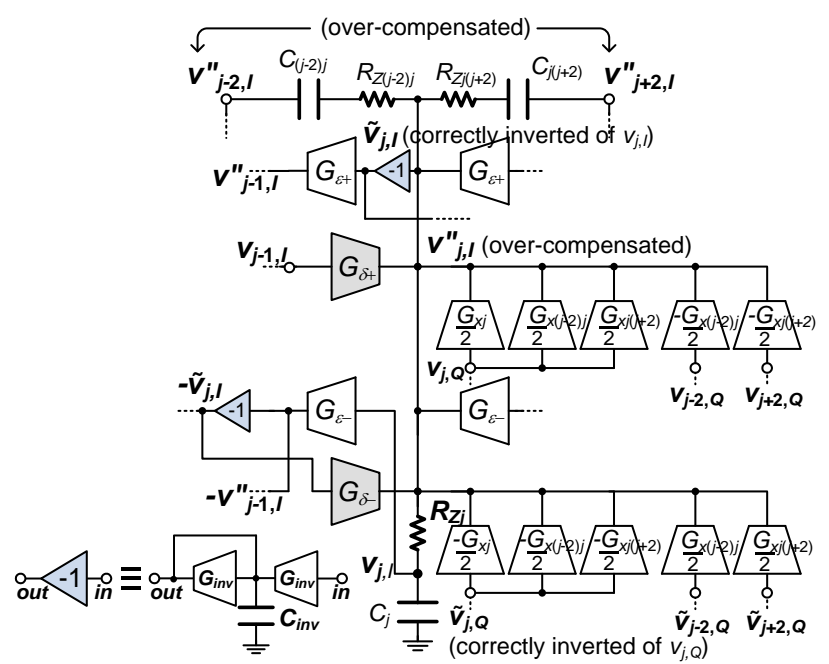

Fig. 12. A DM single-end $I$ section illustrating a pole/zero compensation using $R_{Z}$ in series with capacitors for the proposed SCC filters at the $j^{\text {th }}$ node.

the underlying compensation principle is to make sure that all the transconductors and the floating capacitors in Fig. 3(a) and Fig. 8 supply the current signals into the grounded integrating capacitors $C_{j}$ with no frequency-response distortion. Fig. 12 shows the general schematic at the $j^{\text {th }}$ node of the SCC complex filter's $I$ section (with $\varepsilon_{x}, \delta_{x}=0$ ). Also included in the schematic are the compensation resistors $R_{Z}$ 's in series with the grounded capacitors $C_{j}$ and the floating capacitors $C_{(j-2) j}$, $C_{j(j+2)}$. Following this, the voltage $\tilde{v}_{j}$ at the inverting amplifier's output can be related to the voltage $v_{j}{ }_{j}$ at its input, and the associated current signals injected into $C_{j}$, as given by

$$
\tilde{v}_{j}=\frac{v^{\prime \prime}}{1+s\left(C_{i v v} / G_{i v v}\right)}=\frac{R_{\mathrm{Zj}}+\left(1 / s C_{j}\right)}{1+s\left(C_{i v v} / G_{i v v}\right)}\{\sum_{k} i_{j k}+\overbrace{i_{C(j-2) j}+i_{C(j+2) j}}^{\text {withlating capacitor only }}\}
$$

where $\sum i_{j k}$ represents the sum of the currents from $G_{\varepsilon \pm}, G_{\delta \pm}$, $\pm G_{x j} / 2, \pm G_{x(j-2) j} / 2$ and $\pm G_{x j(j+2)} / 2 ; i_{C(j \pm 2) j}$ are the currents from the floating capacitors $C_{(j-2) j}$ and $C_{j(j+2)}$. Since $C_{(j-2) j}$ and $C_{j(j+2)}$ are connected from the adjacent nodes, $(j-2)^{\text {th }}$, and $(j+2)^{\text {th }}$ to the $j^{\text {th }}$ node, (17) can be rewritten as

$$
\begin{aligned}
& \tilde{v}_{j}=\frac{R_{Z j}+\left(1 / s C_{j}\right)}{1+s\left(C_{i v v} / G_{i v v}\right)}\left\{\sum_{k} i_{j k}+\frac{\left(v^{\prime \prime}{ }_{j-2}-v^{\prime \prime}{ }_{j}\right)}{\left(R_{Z(j-2) j}+\left(1 / s C_{(j-2) j}\right)\right)}+\frac{\left(v^{\prime \prime}{ }_{j+2}-v^{\prime \prime}{ }_{j}\right)}{\left(R_{Z j(j+2)}+\left(1 / s C_{j(j+2)}\right)\right)}\right\}
\end{aligned}
$$

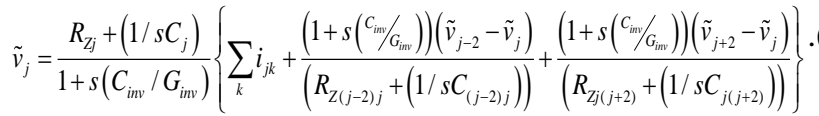

Based on the above equations, it requires that the following conditions be satisfied to achieve perfect compensation:

$$
R_{Z j} C_{j}=R_{Z(j-2) j} C_{(j-2) j}=R_{Z j(j+2)} C_{j(j+2)}=C_{i n v} / G_{i n v} \text {. }
$$

The ideal relation between the input voltages and output currents of the transconductors at $j^{\text {th }}$ node is restored by applying the condition in (19) to (18) where the voltage $\tilde{v}_{j}$ of the associated inverting amplifiers can now be expressed as

$$
\tilde{v}_{j}=\frac{1}{s C_{j}}\{\sum_{k} i_{j k}+\overbrace{s C_{(j-2) j}\left(\tilde{v}_{j-2}-\tilde{v}_{j}\right)+s C_{j(j+2)}\left(\tilde{v}_{j+2}-\tilde{v}_{j}\right)}^{\text {wih flating capacitor only }}\} \text {. }
$$

It is important to note that only the correctly compensated voltage $v_{j}$ or its inverted version $\tilde{v}_{j}$ (not its over-compensated

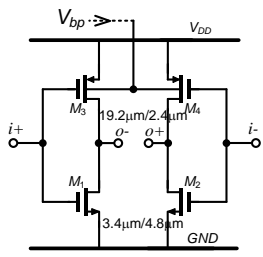

(a) The main $G(\approx 50 \mu \mathrm{S})$

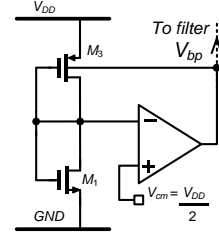

(b) Bias setup circuit generating $V_{b p}$ counterpart, $v_{j}$ ) are used by various transconductors, e.g. $v_{j, I}$ and $\tilde{v}_{j, I}$ are used by $G_{\varepsilon-}$ and $G_{\varepsilon+}$, respectively in Fig. 12. Thus, the compensation resistors $R_{z}$ 's are required only for the grounded capacitors $C_{j}$ and the floating capacitors $C_{i j}$ at the input of the inverting amplifiers.

\section{CiRCUIT Verifications}

The proposed SCC complex filter structure in Fig. 8 has been verified via simulation in a standard $0.18-\mu \mathrm{m}$ CMOS process employing an inverter-based pseudo-differential transconductor from Fig. 5 as the core transconductor cell with minor modification as depicted in Fig. 13(a). In this design, $\alpha$ is selected to be 1.5 to achieve the output noise and the dynamic range (DR) close to that of the conventional design. Since the common-mode $G_{c}$ value is identical to the differential-mode $G$, the value of the DC CM nodal conductance $G_{T j}$ is well-defined where $G_{T j} \approx+2 \varepsilon \delta G_{c}$ for $j=e v e n$ and $G_{T j} \approx+2 G_{c}$ for $j=o d d$ with $\varepsilon_{x}, \delta_{x}=0$ as discussed in Section II.A. Therefore, similar to the technique in [7], [2629], at a fixed body voltage of PMOS, $V_{b p}$, NMOS and PMOS of the transconductor cell can be appropriately sized to set their gate and drain DC bias voltages to $V_{D D} / 2$ for the entire filter without extra circuitry. However, to counter process, temperature and supply voltage variations, a master-slave scheme (e.g. in [23]) is utilized to automatically adjust $V_{b p}$ and accurately set up the required DC bias voltage. This $V_{b p}$ is generated from the master bias set-up circuit of Fig. 13(b) comprising the diode-connected transconductor's DC half circuit in a negative-feedback servo loop. This transconductor's half-circuit replicates the desired DC voltage level of the transconductors working within the filter.

The filter operates at $V_{D D}=1 \mathrm{~V}$ with $1.1-\mathrm{MHz}$ center frequency and $0.8-\mathrm{MHz}$ bandwidth. A stabilization of the SCC filter (with $\varepsilon=1.0, \delta=0.25$ ) is verified in Fig. 14(a) depicting Monte-Carlo (MC) simulations of transient response of the output voltage converge to a stable value of $V_{D D} / 2=0.5 \mathrm{~V}$ after the supply voltage being subjected to a step change from 0 to $1 \mathrm{~V}$ (without input signal). Moreover, the histogram plots in Fig. 14(b) and (c) compare the filter's nodal bias voltages for $\varepsilon_{x}, \delta_{x}=0$ and $\varepsilon_{x}=-\delta_{x}=0.075$ (applied to $G_{x j \varepsilon \pm}, G_{x j \delta \pm}$ at $j=1$, $3,5)$ with the respective means $(\mu)$ and standard deviations $(\sigma)$. As predicted in Section III.A, having $\varepsilon_{x}=-\delta_{x}$ renders the bias voltage levels closer to $V_{D D} / 2$ (especially those at nodes 4). Fig. 15(a) shows the filter's frequency responses with $\varepsilon=$ $0.75,1.0$ and $\delta=0.25$. The $\mathrm{CM}$ frequency response is also included in Fig. 15(a). The in-band CMRR level is at $\sim 43 \mathrm{~dB}$ and it is clear that the DC CM gain decreases with the $\delta / \varepsilon$ ratio where its value is as closely predicted by (8). Fig. 15(b) 


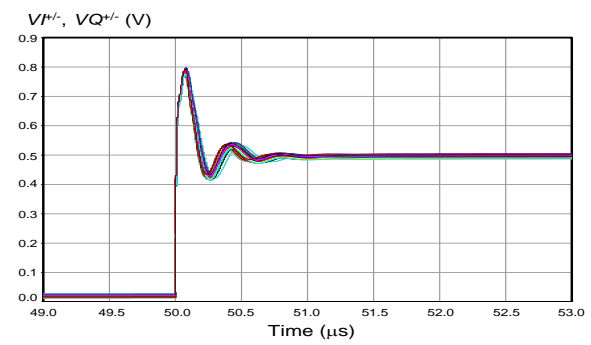

(a) Output voltage response to the $V_{D D}$ step change

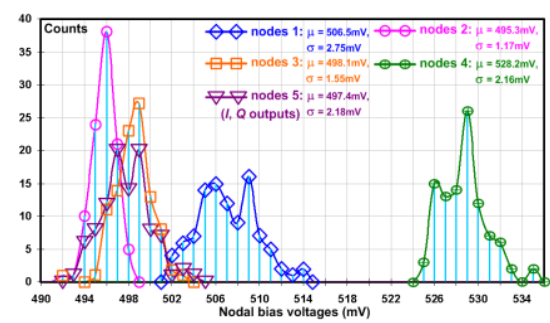

(b) Bias voltage histograms for $\varepsilon_{x}, \delta_{x}=0$

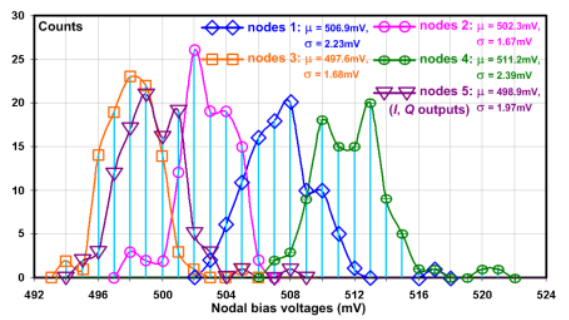

(c) Bias voltage histograms for $\varepsilon_{x}=-\delta_{x}=0.075$ Fig. 14. Monte Carlo simulations (100 runs) to monitor the complex filter's bias voltages

depicts the $\mathrm{MC}$ frequency response indicating that component mismatch plays role in the image-band distorted response.

An two-tone test was carried out with two input frequencies at 1.05 and $1.15 \mathrm{MHz}$. Fig. 16 shows an in-band intermodulation (IM) level plot where the IIP3 values are at $+3 \mathrm{dBV}_{\mathrm{rms}}$ for the SCC filter and $+1 \mathrm{dBV}_{\mathrm{rms}}$ for the conventional filter. The output noise power spectral density is shown in Fig. 17 with the output noise levels (integrated from $10 \mathrm{kHz}$ to $10 \mathrm{MHz}$ ) of $6.4 \times 10^{-9} \mathrm{~V}^{2}$ and $6.1 \times 10^{-9} \mathrm{~V}^{2}$ for the SCC and conventional filters, respectively. With the filter's passband gain of 0.5 , these output noise could be respectively translated to an input-referred noise of $-75.92 \mathrm{dBV}_{\text {rms }}$ and $76.13 \mathrm{dBV}_{\text {rms. }}$ The IM test also indicates a linear gain up to an input level of $-13.0 \mathrm{dBV}_{\mathrm{rms}}$ for both filters - with the corresponding signal-to-noise ratios (SNR) of $62.9 \mathrm{~dB}$ and 63.1dB. Following the calculation in [2], [7], where the spurious-free dynamic range, $\mathrm{SFDR}_{\mathrm{dB}}=(2 / 3)\left(\mathrm{IIP} 3_{\mathrm{dBV}} \mathrm{rms}-\right.$ InputNoise $_{\mathrm{dBV}} \mathrm{rms}_{\mathrm{r}}$, the in-band SFDRs have been calculated at $52.6 \mathrm{~dB}$ (the SCC) and $51.4 \mathrm{~dB}$ (the conventional). The out-ofband IM was also simulated with two blocker frequencies at 3 and $4 \mathrm{MHz}$ where the corresponding SFDRs have been found at $56.3 \mathrm{~dB}$ (the SCC) and $56.8 \mathrm{~dB}$ (the conventional).

The conventional and proposed complex filters consume static power of $851 \mu \mathrm{W}$ and $549 \mu \mathrm{W}$, respectively. The SCC filter prototype thus renders $O P R \approx 3.4$ or $70.5 \%$ saving on the overhead power consumption with no significant deterioration on the DR. This $O P R$ value is closely agreed with the calculated of 3.7 from (13) using $\omega_{b} / \omega_{0}=(0.88 / 2) / 1.1$ $=2.5, \overline{C_{x f}} / \overline{C_{x}} \approx 0.23$, and $\alpha=1.5$ for this particular design. As summarized in Table III, the filters have been compared using a figure-of-merit, $\mathrm{FoM}=$ (Power Consumption) $) /\left(N \cdot f_{c} \cdot D R\right)$ where $f_{c}$ is the centre operating frequency. The calculation suggests that the SCC filter renders a significant improvement by $40 \%$ and $30 \%$ on the FoM respectively with the in-band and out-of-band SFDR.

\section{CONCLUSION}

A low-power $G_{m^{-}} C$ filter structure with self CM stabilization and $\mathrm{CM}$ rejection has been proposed. Through the use of inverting amplifiers, the proposed structure removes all the $\mathrm{CM}$ positive feedback loops and achieves stabilization without employing a dedicated, power-hungry $\mathrm{CM}$ control network usually utilized in the conventional design. The filter's CM conductance, CM gain, frequency and noise responses have been analyzed to assist the design. With no performance degradation in terms of in-band and out-of-band SFDR or SNR, the self-CM-controlled $5^{\text {th }}$-order elliptic complex filter offers over $70 \%$ saving on the overhead power compared to the conventional design. Although the proposed

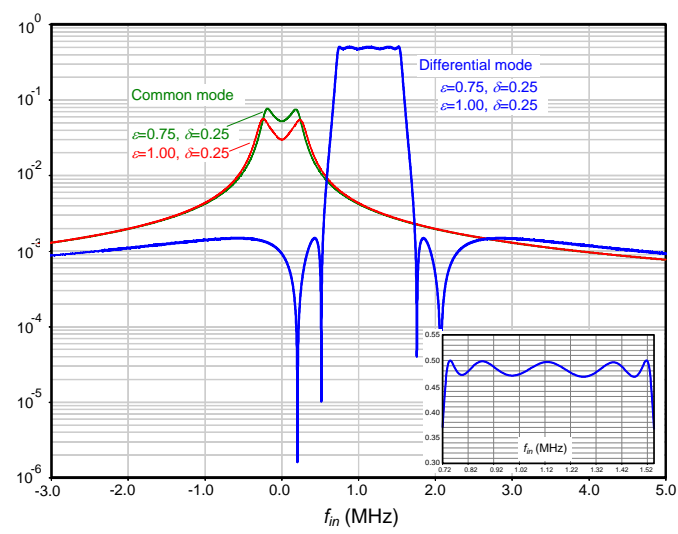

(a) DM and CM frequency responses

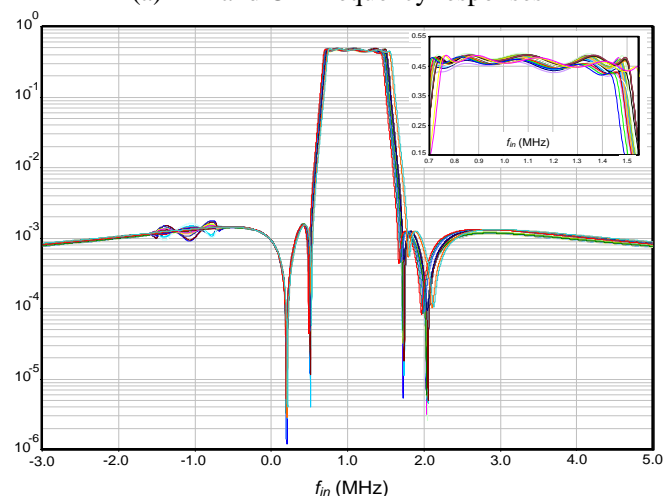

(b) DM frequency response with Monte-Carlo analysis Fig. 15. The SCC complex filter's frequency response

$G_{m}-C$ filter has been demonstrated with the inverter-based pseudo-differential transconductor, the concept can be applied to a vast range of low-voltage transconductors - especially those without intrinsic capability to reject CM signals.

\section{REFERENCES}

[1] B. Razavi, RF Microelectronics, $2^{\text {nd }}$ ed., Prentice Hall, 2011.

[2] T. H. Lee, The Design of CMOS Radio-Frequency Integrated Circuits, $2^{\text {nd }}$ ed., Cambridge University Press, Cambridge, U.K., 2004.

[3] J. Crols and M. S. J. Steyaert "Low-IF Topologies for High-Performance Analog Front Ends of Fully Integrated Receivers," IEEE Trans. Circuits Syst. -II, vol. 45, no. 3, March 1998.

[4] S. Tadjpour et al., "A 900-MHz Dual-Conversion Low-IF GSM Receiver in 0.35- $\mu \mathrm{m}$ CMOS," IEEE J. Solid-State Circuits, vol. 36, no. 12, Dec. 2001. [5] K. W. Martin, "Complex Signal Processing Is Not Complex," IEEE Trans. Circuits Syst. I, vol. 51, no. 9, Sep. 2004.

[6] A. Amira and E. Sánchez-Sinencio, "A Pseudo Differential Complex Filter for Bluetooth With Frequency Tuning," IEEE Trans. Circuits Syst. -II, vol. 50 , no. 10 , Oct. 2003.

[7] B. Guthrie, J. Hughes, T. Sayers, and A. Spencer, "A CMOS Gyrator Low-IF Filter for a Dual-Mode Bluetooth/ZigBee Transceiver," IEEE $J$. Solid-State Circuits, vol. 40, Sep. 2005.

[8] J. Ko, J. Kim, S. Cho, and K. Lee, "A 19-mW 2.6-mm² L1/L2 dual-band CMOS GPS receive,” IEEE J. Solid-State Circuits, Jul. 2005. 
TABLE III PERFORMANCE COMPARISON IN 0.18- $\mu \mathrm{M}$ CMOS

\begin{tabular}{|l|c|c|}
\hline Filter type, $V_{D D}$ & \multicolumn{2}{|c|}{ Complex $5^{\text {th }}$-order elliptic, $1 \mathrm{~V}$} \\
\hline Total integrating capacitance & Conventional & SCC \\
\hline Total transconductance & $151.74 \mathrm{pF}$ & $151.74 \mathrm{pF}$ \\
\hline Power consumption, $O P R$ & $4198.29 \mu \mathrm{S}$ & $2649.14 \mu \mathrm{S}$ \\
\hline Input-referred noise & $851 \mu \mathrm{W}, 1$ & $549 \mu \mathrm{W}, 3.4$ \\
\hline Signal-to-noise ratio (SNR) & $-76.13 \mathrm{dBV}$ rms & $-75.92 \mathrm{dBV}_{\text {rms }}$ \\
\hline In-band DR: SFDR & $63.1 \mathrm{~dB}$ & $62.9 \mathrm{~dB}$ \\
IIP3-Input Noise & $51.4 \mathrm{~dB}$ & $52.6 \mathrm{~dB}$ \\
\hline Out-of-band DR: SFDR & $77.1 \mathrm{~dB}$ & $78.9 \mathrm{~dB}$ \\
\hline IIP3 - Input Noise & $56.8 \mathrm{~dB}$ & $56.3 \mathrm{~dB}$ \\
FoM based on: In-band SFDR & $0.1 \mathrm{~dB}$ & $84.4 \mathrm{~dB}$ \\
Out-of-band SFDR & $0.11 \mathrm{pJ}$ & $0.12 \mathrm{pJ}$ \\
\hline
\end{tabular}

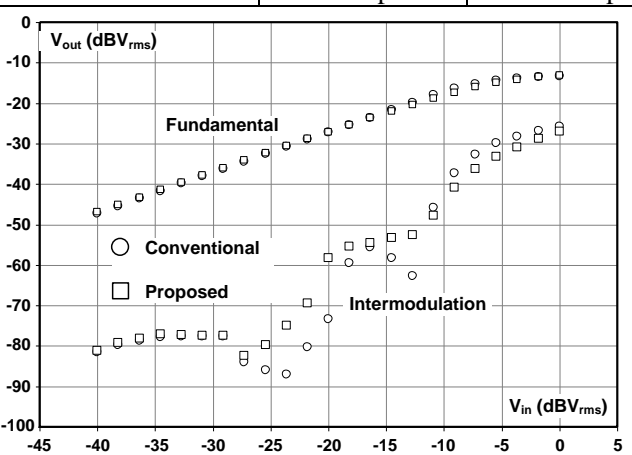

Fig. 16. In-band two-tone intermodulation test

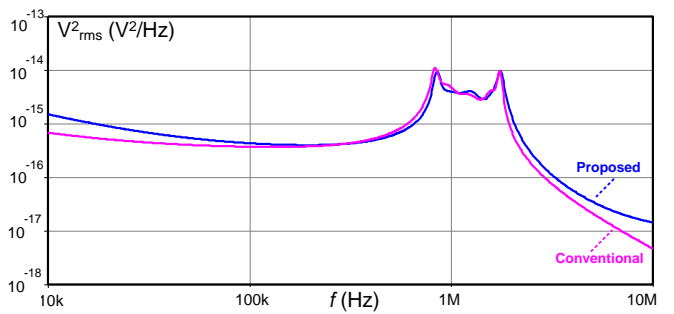

Fig. 17. Output noise power spectral density

[9] I. Nam et al., "A 2.4-GHz Low-Power Low-IF Receiver and DirectConversion Transmitter in $0.18-\mu \mathrm{m}$ CMOS for IEEE 802.15.4 WPAN Applications," IEEE Trans. Microw. Theory Techn., Vol.55, no.4, Apr. 2007. [10] R. Chen and H. Hashemi, "Reconfigurable Receiver With RadioFrequency Current-Mode Complex Signal Processing Supporting Carrier Aggregation," IEEE J. Solid-State Circuits, vol. 50, no. 12, Dec. 2015.

[11] A. Selvakumar, M. Zargham, and A. Liscidini, "Sub-mW Current Re-Use Receiver Front-End for Wireless Sensor Network Applications," IEEE J. Solid-State Circuits, vol. 50, no. 12, Dec. 2015.

[12] X. Yu et al., "A Fully-Integrated Reconfigurable Dual-Band Transceiver for Short Range Wireless Communications in $180 \mathrm{~nm}$ CMOS," IEEE J. SolidState Circuits, vol. 50, no. 11, November, 2015.

[13] B. Liu, "Receiver Architecture Employing Low Intermediate Frequency and Complex Filtering," U.S. Patent 7545 857, June, 9, 2009.

[14] Y. P. Tsividis and J. O. Voorman, Integrated Continuous-Time Filters. Piscataway, NJ: IEEE Press, 1993.

[15] Y. Sun (editor), Design of High Frequency Integrated Analogue Filters, IET, 2002.

[16] E. A. M. Klumperink "Systematic Comparison of HF CMOS Transconductors," IEEE Trans. Circuits Syst. -II, vol.50, no.10, Oct. 2003.

[17] F. Behbahani, W. Tan, A. Karimi-Sanjaani, A. Roithmeier, and A. A. Abidi, "A Broad-Band Tunable CMOS Channel-Select Filter for a Low-IF Wireless Receiver," IEEE J. Solid-State Circuits, vol.35, Apr. 2000.

[18] A. Baschirotto, F. Rezzi, and R. Castello, "A low-voltage balanced transconductor with high input common-mode rejection," IET. Electronics Letters, vol. 30, no. 20, pp. 1669-1671, Sept. 29, 1994.

[19] F. Rezzi, A. Baschirotto, and R. Castello, "A 3 V 12-55 MHz BiCMOS Pseudo-Differential Continuous-Time Filter," IEEE Trans. Circuits Syst.-I, vol. 42, no. 11, Nov. 1995.

[20] V. Saari, M. Kaltiokallio, S. Lindfors, J. Ryynanen, K.A.I. Halonen, "A $240-\mathrm{MHz}$ low-pass filter with variable gain in 65-nm CMOS for a UWB radio receiver," IEEE Trans. Circuits Syst. -I, vol.56, no.7, Jul. 2008.
[21] P. Khumsat et al., "Differential-Mode/Common-Mode Feedforward Transconductor for Low-Voltage $\mathrm{G}_{\mathrm{m}}-\mathrm{C}$ filters," The Proc. of IEEE Int. Conf. on Electronics, Circuits and Systems, 10-13 Dec. 2006.

[22] H. Tanimoto and M. Katakura, "Fully-Differential Amplifier," U. S. Patent 7619 473, Nov. 17, 2009.

[23] P. Khumsat and A. Worapishet, "A 0.5-V R-MOSFET-C Filter Design Using Subthreshold R-MOSFET Resistors and OTAs With Cross-Forward Common-Mode Cancellation Technique," IEEE J. Solid-State Circuits, vol. 47, Nov. 2012.

[24] Z. Lin et al., "A $1.7 \mathrm{~mW} 0.22 \mathrm{~mm}^{2} 2.4 \mathrm{GHz}$ ZigBee RX exploiting a current-reuse blixer and hybrid filter topology in $65 \mathrm{~nm}$ CMOS," in Proc. IEEE Int. Solid-State Circuits Conf. Dig. Tech. Papers, Feb. 2013.

[25] A. Selvakumar and A. Liscidini, "Current-Recycling Complex Filter for Bluetooth-Low-Energy Applications," IEEE Trans. Circuits Syst. -II, vol.62, no.4, pp.332-336, April, 2015.

[26] B. Nauta and E. Seevinck, "Linear CMOS transconductance element for VHF filters," Electronics Letters, vol. 25, no. 7, 1989.

[27] B. Nauta, "CMOS VHF transconductance-C lowpass filter," Electronics Letters, vol. 26, no. 7, 1990.

[28] B. Nauta, "A CMOS Transconductance-C Filter Technique For Very High Frequencies," IEEE J. Solid-State Circuits, vol.27, Feb. 1992.

[29] P. Andreani and S. Mattisson, "On the Use of Nauta's Transconductor in Low-Frequency CMOS $g_{m}-C$ Bandpass Filters," IEEE J. Solid-State Circuits, vol. 37, pp. 114-124, Feb. 2002.

[30] J.B. Hughes, A. Spencer, A. Worapishet and R. Sitdhikorn,"1 mW CMOS polyphase channel filter for Bluetooth," IEE Proceedings - Circuits, Devices and Systems, vol. 149, no. 56, 2002.

[31] P. Crombez, J. Craninckx, and M. Steyaert, "A $100 \mathrm{kHz}-20 \mathrm{MHz}$ Reconfigurable Nauta $g_{m}-C$ Biquad Lowpass Filter in $13 \mu \mathrm{m}$ CMOS," Proc. IEEE Asian Solid-State Conference, Nov. 12-14, 2007.

[32] J. Mahattanakul, P. Khumsat, and W. Surakampontorn, "Selection of the common-mode feedback network connection of fully differential $\mathrm{G}_{\mathrm{m}}-\mathrm{C}$ filters," IET Circuits, Devices \& Systems, vol.3, no. 1, 2009.

[33] J.B. Hughes, "Balanced Transconductor and Electronic Device," U. S. Patent 6680 627, Jan. 20, 2004.

[34] J.W. Park and C. S. Kim, "Nauta Operational Transconductance Amplifier," U. S. Patent 7616 056, November, 10, 2009.

[35] T.-Y. Lo and C.-C. Hung, "A 1 GHz Equiripple Low-Pass Filter With a High-Speed Automatic Tuning Scheme," IEEE Trans. Very Large Scale Integr. (VLSI) Syst, vol. 19, no 2, Feb. 2011, pp. 175-181.

[36] M. Darvishi, R. van der Zee, B. Nauta, "Design of Active N-Path Filters," IEEE J. Solid-State Circuits, vol.48, no.12, Dec. 2013.

[37] H. Kundur Subramaniyan et al., "RF Transconductor Linearization Robust to Process, Voltage and Temperature Variations," IEEE J. Solid-State Circuits, vol.50, Nov. 2015.

[38] A. Wyszynski and R. Schaumann, "Avoiding common-mode feedback in continuous-time $\mathrm{g}_{\mathrm{m}}$ - C filters by use of lossy integrators," in Proc. IEEE Int. Symp. Circuits Syst., vol.5, pp. 281-284, 1994.

[39] T. Choogorn and J. Mahattanakul, "Relationship between common-mode rejection and differential-mode distortion in fully differential $\mathrm{G}_{\mathrm{m}}-\mathrm{C}$ filters," IET Circuits, Devices \& Systems, vol.5, no.6, Nov. 2011.

[40] J. Mahattanakul and P. Khumsat, "Structure of complex elliptic $\mathrm{G}_{\mathrm{m}}-\mathrm{C}$ filters suitable for fully differential implementation," IET Circuits, Devices \& Systems, vol. 1, no. 4, August, 2007, pp. 275 - 282.

[41] S. Kousai, M. Hamada, R. Ito, and T. Itakura, "A 19.7 MHz, Fifth-Order Active-RC Chebyshev LPF for Draft IEEE802.11n With Automatic QualityFactor Tuning Scheme" IEEE J. Solid-State Circuits, Nov. 2007.

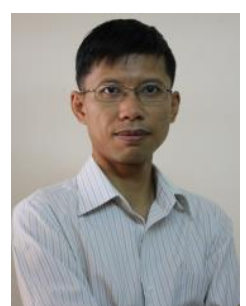

Phanumas Khumsat (M'05) Phanumas Khumsat received his M.Eng. in 1997 and $\mathrm{Ph} . \mathrm{D}$. in 2002 both in Electrical and Electronic Engineering from Imperial College London, UK. Since 2003, he has been with the Department of Electrical Engineering, Faculty of Engineering, Prince of Songkla University, Hat-Yai, Thailand where he is now serving as an Associate Professor. His research interests include analog integrated circuits in CMOS and BiCMOS technologies for wireless radio and optical communications, biomedical and agriculture applications. He is now serving as an associate editor for IET Circuits, Devices \& Systems. 